\title{
Holonomy Groups and Algebras
}

\author{
Lorenz J. Schwachhöfer
}

Preprint 2010-06

April 2010

Fakultät für Mathematik

Technische Universität Dortmund

Vogelpothsweg 87

44227 Dortmund

$\underline{\text { tu-dortmund.de/MathPreprints }}$ 



\title{
Holonomy Groups and Algebras
}

\author{
Lorenz J. Schwachhöfer*
}

April 9, 2010

\section{Introduction}

An affine connection is one of the basic objects of interest in differential geometry. It provides a simple and invariant way of transferring information from one point of a connected manifold $M$ to another and, not surprisingly, enjoys lots of applications in many branches of mathematics, physics and mechanics. Among the most informative characteristics of an affine connection is its holonomy group which is defined as the subgroup $\operatorname{Hol}_{p}(M) \subset \operatorname{Aut}\left(T_{p} M\right)$ consisting of all automorphisms of the tangent space $T_{p} M$ at $p \in M$ induced by parallel translations along $p$-based loops.

The notion of holonomy first arose in classical mechanics at the end of the 19th century. It was Heinrich Hertz who used the terms 'holonomic' and 'non-holonomic' constraints in his magnum opus Die Prinzipien der Mechanik, in neuen Zusammenhängen dargestellt ("The principles of mechanics presented in a new form") which appeared one year after his death in 1895. For a more detailed exposition of the early origins of the holonomy problem, see also [21].

The notion of holonomy in the mathematical context seems to have appeared for the first time in the work of E.Cartan $([30,31,33])$. He considered the Levi-Civita connection of a Riemannian manifold $M$, so that the holonomy group is contained in the orthogonal group. He showed that in this case, the holonomy group is always connected if $M$ is simply connected. Moreover, he observed that $\operatorname{Hol}_{p}(M)$ and $\operatorname{Hol}_{q}(M)$ are conjugate via parallel translation along any path from $p$ to $q$, hence the holonomy group $\operatorname{Hol}(M) \subset G l(n, \mathbb{R})$ is well defined up to conjugation.

Cartan's interest in holonomy groups was due to his observation that for a Riemannian symmetric space, the holonomy group and the isotropy group coincide up to connected components, as long as the symmetric space contains no Euclidean factor. This insight he used to classify Riemannian symmetric spaces ([32]).

In the 1950s, the concept of holonomy groups was treated more thoroughly. In 1952, Borel and Lichnerowicz ([14]) proved that the holonomy group of a Riemannian manifold is always a Lie subgroup, possibly with infinitely many components. In the same year, de Rham ([37]) proved what is nowadays called the de Rham Splitting theorem. Namely, if the holonomy of a Riemannian manifold is reducible, then the metric must be a local product metric; if the manifold is in addition

*Fakultät für Mathematik, Technische Universität Dortmund, Vogelpothsweg 87, 44221 Dortmund, Germany; email: 1schwach@math.uni-dortmund.de 
complete and simply connected, then it must be a Riemannian product globally. In 1954, Ambrose and Singer proved a result relating the Lie algebra of the holonomy group and the curvature map of the connection ([2]).

A further milestone was reached by M.Berger in his doctoral thesis ([9]). Based on the theorem of Ambrose and Singer, he established necessary conditions for a Lie algebra $\mathfrak{g} \subset \operatorname{End}(V)$ to be the Lie algebra of the holonomy group of a torsion free connection, and used it to classify all irreducible non-symmetric holonomy algebras of Riemannian metrics, i.e., such that $\mathfrak{g} \subset \mathfrak{s o}(n)$. The list is remarkably short. In fact, it is included in (and almost coincides with) the list of connected linear groups acting transitively on the unit sphere. This fact was proven later directly by J.Simons ([66]) in an algebraic way. Recently, C.Olmos gave a beautiful simple argument showing this transitivity using elementary arguments from submanifold theory only ([59]).

Together with his list of possible Riemannian holonomy groups, Berger also gave a list of possible irreducible holonomy groups of pseudo-Riemannian manifolds, i.e., manifolds with a nondegenerate metric which is not necessarily positive definite. Furthermore, in 1957 he generalized Cartan's classification of Riemannian symmetric spaces to the isotropy irreducible ones ([10]).

In the beginning, it was not clear at all if the entries on Berger's list occur as the holonomy group of a Riemannian manifold. In fact, it took several decades until the last remaining cases were shown to occur by Bryant ([16]). As it turns out, the geometry of manifolds with special holonomy groups are of utmost importance in many areas of differential geometry, algebraic geometry and mathematical physics, in particular in string theory. It would lead to far to explain all of these here, but rather we refer the reader to [11] for an overview of the geometric significance of these holonomies.

In 1998, S.Merkulov and this author classified all irreducible holonomy groups of torsion free connections ([56]). In the course of this classification, some new holonomies were discovered which are symplectic, i.e., they are defined on a symplectic manifold such that the symplectic form is parallel. The first such symplectic example was found by Bryant ([17]); later, in ([34, 35]) an infinite family of such connection was given. These symplectic holonomies share some striking rigidity properties which later were explained on a more conceptual level by M.Cahen and this author ([26]), linking them to parabolic contact geometry.

In this article, we shall put the main emphasis on the investigation of connections on principal bundles as all other connections can be deduced from these. This allows us to prove most of the basic results in greater generality than they were originally stated and proven. Thus, section 2 is devoted to the collection of the basic definitions and statements, where in most cases, sketches of the proofs are provided. In section 3, we shall collect the known classification results where we do not say much about the proofs, and finally, in section 4 we shall describe the link of special symplectic connections with parabolic contact geometry. 


\section{Basic definitions and results}

\subsection{Connections on principal bundles}

Let $\pi: P \rightarrow M$ be a (right)-principal $G$-bundle, where $M$ is a connected manifold and $G$ is a Lie group with Lie algebra $\mathfrak{g}$. A principal connection on $P$ may be defined as a $\mathfrak{g}$ - valued one-form $\omega \in \Omega^{1}(P) \otimes \mathfrak{g}$ such that

1. $\omega$ is $G$-equivariant, i.e., $r_{g^{-1}}^{*}(\omega)=A d_{g} \circ \omega$ for all $g \in G$,

2. $\omega\left(\xi^{*}\right)=\xi$ for all $\xi \in \mathfrak{g}$, where $\xi_{p}^{*}:=\left.\frac{\mathrm{d}}{\mathrm{d} t}\right|_{t=0}(p \cdot \exp (t \xi))$ denotes the action field corresponding to $\xi$.

Here, $r_{g}: P \rightarrow P$ denotes the right action of $G$. Alternatively, we may define a principal connection to be a $G$-invariant splitting of the tangent bundle

$$
T P=\mathscr{H} \oplus \mathscr{V}, \text { where } \mathscr{V}_{p}=\operatorname{ker}(d \pi)_{p}=\operatorname{span}\left(\left\{\xi_{p}^{*} \mid \xi \in \mathfrak{g}\right\}\right) \text { for all } p \in P
$$

In this case, $\mathscr{H}$ and $\mathscr{V}$ are called the vertical and horizontal space, respectively.

To see that these two definitions are indeed equivalent, note that for a given connection oneform $\omega \in \Omega^{1}(P) \otimes \mathfrak{g}$, we may define $\mathscr{H}:=\operatorname{ker}(\omega)$; conversely, given the splitting (1), we define $\omega$ by $\left.\omega\right|_{\mathscr{H}} \equiv 0$ and $\omega\left(\xi^{*}\right)=\xi$ for all $\xi \in \mathfrak{g}$; it is straightforward to verify that this establishes indeed a one-to-one correspondence.

The curvature form of a principal connection is defined as

$$
\Omega:=\mathrm{d} \omega+\frac{1}{2}[\omega, \omega] \in \Omega^{2}(P) \otimes \mathfrak{g} .
$$

For its exterior derivative we get

$$
\mathrm{d} \Omega+[\omega, \Omega]=0 .
$$

By the Maurer-Cartan equations, it follows from (2) that

$$
\left.\xi^{*}\right\lrcorner \Omega=0 \text { for all } \xi \in \mathfrak{g} \text {, and } \mathrm{d} r_{g}^{*}(\Omega)=A d_{g} \circ \Omega \text {. }
$$

A (piecewise smooth) curve $c:[a, b] \rightarrow P$ is called horizontal if $c^{\prime}(t) \in \mathscr{H}_{c(t)}$ for all $t \in[a, b]$. Evidently, for every curve $\underline{c}:[a, b] \rightarrow M$ and $p \in \pi^{-1}(\underline{c}(a))$, there is a unique horizontal curve $c^{p}:[a, b] \rightarrow P$, called horizontal lift of $c$, with $\underline{c}=\pi \circ c^{p}$ and $c^{p}(a)=p$. Since by the $G$-equivariance of $\mathscr{H}$ we have $c^{p \cdot g}=r_{g} \circ c^{p}$, the correspondence

$$
\Pi_{\underline{c}}: \pi^{-1}(\underline{c}(a)) \longrightarrow \pi^{-1}(\underline{c}(b)), \quad p \longmapsto c^{p}(b)
$$

is $G$-equivariant and is called parallel translation along $\underline{c}$. The holonomy at $p \in P$ is then defined as

$$
H o l_{p}:=\left\{g \in G \mid p \cdot g=\Pi_{\underline{c}}(p) \text { for } \underline{c}:[a, b] \rightarrow M \text { with } \underline{c}(a)=\underline{c}(b)=\pi(p)\right\} \subset G \text {. }
$$


Evidently, $\mathrm{Hol}_{p} \subset G$ is a subgroup as we can concatenate and invert loops. Also, the $G$ - equivariance of $\mathscr{H}$ implies that

$$
\mathrm{Hol}_{p \cdot g}=g^{-1} \mathrm{Hol}_{p} \mathrm{~g} \text {. }
$$

Moreover, if we pick any path $\underline{c}:[a, b] \rightarrow M$ then, again by concatenating paths, we obtain for $p \in \pi^{-1}(\underline{c}(a))$

$$
\mathrm{Hol}_{\Pi_{\underline{c}}(p)}=\mathrm{Hol}_{p} .
$$

Thus, by (6) and (7) it follows that the holonomy group $\mathrm{Hol} \cong \mathrm{Hol}_{p} \subset G$ is well defined up to conjugation in $G$, independent of the choice of $p \in P$.

We define the equivalence relation $\sim$ on $P$ by saying that

$$
p \sim q \text { if } p \text { and } q \text { can be joined by a horizontal path. }
$$

Then definition (5) can be equivalently formulated as

$$
H_{p} l_{p}:=\{g \in G \mid p \cdot g \sim p\} .
$$

Theorem 2.1 (Ambrose-Singer-Holonomy Theorem [2]) Let $\pi: P \rightarrow M$ be a principal G-bundle with a connection $\omega \in \Omega^{1}(P) \otimes \mathfrak{g}$ and the corresponding horizontal distribution $\mathscr{H} \subset T P$.

1. The smallest involutive distribution on $P$ which contains $\mathscr{H}$ is the distribution

$$
\hat{\mathscr{H}}_{p}:=\mathscr{H}_{p} \oplus\left\{\xi_{p}^{*} \mid \xi \in \mathfrak{h o l}_{p}\right\}
$$

where $\mathfrak{h o l}_{p} \subset \mathfrak{g}$ is the Lie subalgebra generated by

$$
\mathfrak{h o l}_{p}=\left\langle\left\{\Omega\left(d \Pi_{\underline{c}}(v), d \Pi_{\underline{c}}(w)\right) \mid v, w \in T_{p} P, \underline{c}:[a, b] \rightarrow M \text { any path with } \underline{c}(a)=\pi(p)\right\}\right\rangle .
$$

2. The identity component of $\left(\mathrm{Hol}_{p}\right)_{0} \subset G$ is a (possibly non-regular) Lie subgroup with Lie algebra $\mathfrak{h o l}_{p}$.

Proof. Observe first that the dimension of the right hand side of (10) is independent of $p \in P$. Indeed, from the definition, $\hat{\mathscr{H}}_{q \cdot g}=\mathrm{d} r_{g}\left(\hat{\mathscr{H}}_{q}\right)$, so that this dimension is independent of the point in the fiber of $P$; moreover, if $p \sim q$ and $\underline{c}:[a, b] \rightarrow M$ is a path with horizontal lift joining $p$ and $q$, then it follows from the very definition that $\hat{\mathscr{H}}_{q} \cap \mathscr{V}_{q}=\mathrm{d} \Pi_{\underline{c}}\left(\hat{\mathscr{H}}_{p} \cap \mathscr{V}_{p}\right)$, and $\mathrm{d} \Pi_{\underline{c}}$ is an isomorphism.

To see that $\hat{\mathscr{H}}$ is involutive, let $\underline{X}, \underline{Y} \in \mathscr{X}(M)$ be vector fields and $X, Y \in \mathscr{X}(P)$ be their horizontal lifts. Note that the flows $\Phi_{X}^{t}$ and $\Phi_{\underline{X}}^{t}$ relate as

$$
\Phi_{X}^{t}=\Pi_{\underline{c}_{\underline{X}}^{t}}, \text { where } \underline{c}_{\underline{X}}^{t}:[0, t] \rightarrow M \text { is a trajectory of } \underline{X} \text {. }
$$

Therefore, if we let $\hat{\mathscr{V}}_{p}:=\left\{\xi_{p}^{*} \mid \xi \in \mathfrak{h o l} \mathfrak{l}_{p}\right\}$, then the definition of $\mathfrak{h o l} \mathfrak{l}_{p}$ implies that $\Phi_{X}^{t}\left(\hat{\mathscr{V}}_{p}\right)=\hat{\mathscr{V}}_{q}$, where $q=\Phi_{X}^{t}(p)$ and thus, $\left[X, \hat{\mathscr{V}}_{p}\right] \subset \hat{\mathscr{V}}_{p}$ for all horizontal vector fields $X$, i.e., $[\mathscr{H}, \hat{\mathscr{V}}] \subset \hat{\mathscr{H}}$.

Next, by (2), $[X, Y]=-\xi_{\Omega(X, Y)}^{*} \bmod \mathscr{H}$ for all horizontal vector fields $X, Y$ so that $[\mathscr{H}, \mathscr{H}] \subset$ $\hat{\mathscr{H}}$; finally, $[\hat{\mathscr{V}}, \hat{\mathscr{V}}] \subset \hat{\mathscr{V}}$ as $\mathfrak{h o l}_{p}$ is a Lie algebra by definition. 
Thus, $\hat{\mathscr{H}} \subset P$ is an involutive distribution. Conversely, the above arguments show that any involutive distribution containing $\mathscr{H}$ also contains $\hat{\mathscr{H}}$, so that $\hat{\mathscr{H}}$ is minimal as asserted.

Let $P_{0} \subset P$ be a maximal leaf of $\hat{\mathscr{H}}$, let $p_{0} \in P_{0}$ and let

$$
H:=\left\{g \in G \mid p_{0} \cdot g \in P_{0}\right\} \subset G .
$$

Since $\mathscr{H}$ and hence $\hat{\mathscr{H}}$ is $G$-invariant, it follows that $H \subset G$ is a subgroup. In fact, $H \subset G$ is a (possibly non-regular) Lie subgroup since $H \cong P_{0} \cap \pi^{-1}\left(\pi\left(p_{0}\right)\right)$. In fact, the restriction $\pi: P_{0} \rightarrow M$ is a principal $H$-bundle.

Standard arguments now show that $P_{0}$ is indeed a single equivalence class w.r.t. $\sim$, so that $H=H_{o l}$ is a Lie subgroup of $G$ with Lie algebra $\mathfrak{h o l}_{p}$. See e.g. [4] for details.

Definition 2.2 Let $P \rightarrow M$ be a principal $G$-bundle, and let $H \subset G$ be a (possibly non-regular) Lie subgroup of $G$. We call a (possibly non-regular) submanifold $P^{\prime} \subset P$ an $H$-reduction of $P$ if the restriction $\pi: P^{\prime} \rightarrow M$ is a principal $H$-bundle.

In particular, a maximal leaf $P_{0} \subset P$ of the distribution $\hat{\mathscr{H}}$ from Theorem 2.1 is called a holonomy reduction of $P$ which is therefore a reduction with structure group $\mathrm{Hol} \subset \mathrm{G}$. We denote the restriction of $\omega, \Omega$ and $\mathscr{H}$ to $P_{0}$ by the same symbols.

By the $G$-equivariance of the distributions $\mathscr{H}$ and $\hat{\mathscr{H}}$ it follows that if $P_{0}, P_{0}^{\prime} \subset P$ are two holonomy reductions then $P_{0}^{\prime}=r_{g}\left(P_{0}\right)$ for some $g \in G$. That is, the holonomy reduction $P_{0} \subset P$ is unique up to the right $G$-action, and this allows to speak of the holonomy reduction.

The connection $\omega$ is called locally flat if $\Omega=0$. By the above definitions, $\Omega=0$ if and only if the horizontal distribution $\mathscr{H}$ from (1) is involutive, hence the holonomy reduction $P_{0} \rightarrow M$ is a regular covering with deck group $\mathrm{Hol}$. It follows that the pull-back of this covering, $\left(\left.\pi\right|_{P_{0}}\right)^{*}(P)=$ $P_{0} \times G$, is the trivial bundle, and $\omega$ is simply the pull back of the Maurer-Cartan form on $G$ under projection onto the second factor.

This idea can be generalized as follows.

Proposition 2.3 Let $\pi: P \rightarrow M$ be a principal G-bundle with a connection with holonomy group $\mathrm{Hol} \subset \mathrm{G}$, and let $\mathrm{Hol}^{0} \subset \mathrm{Hol}$ denote the identity component. Then there is a regular covering $p: \tilde{M} \rightarrow M$ with deck group $\Gamma:=\mathrm{Hol}_{/} \mathrm{Hol}^{0}$ such that the pull-back bundle $\mathrm{p}^{*}(\mathrm{P}) \rightarrow \tilde{M}$ with the connection $\mathrm{p}^{*}(\omega)$ has holonomy group $\mathrm{Hol}^{0}$.

In particular, if $M$ is simply connected, then the holonomy group of any connection on $P \rightarrow M$ is connected.

Proof. Let $P_{0} \subset P$ be a holonomy reduction, and let $\tilde{M}:=P_{0} / \mathrm{Hol}^{0}$. Then the induced map $p: \tilde{M} \rightarrow$ $M$ is a principal $\Gamma$-bundle, and since $\Gamma$ is discrete, it follows that $p$ is a regular covering. Thus, we have the commutative diagram of principal bundles

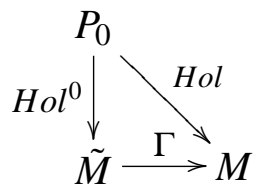


with the indicated structure groups, and the distribution $\mathscr{H}$ on $P_{0}$ induces a connection on each of the principal bundles indicated by the vertical arrows. It follows now that $P_{0} \rightarrow \tilde{M}$ is the holonomy reduction of $p^{*}\left(P_{0}\right) \rightarrow \tilde{M}$.

The regular covering $p: \tilde{M} \rightarrow M$ yields a short exact sequence

$$
0 \longrightarrow \pi_{1}(\tilde{M}) \stackrel{p_{*}}{\longrightarrow} \pi_{1}(M) \stackrel{m}{\longrightarrow} \Gamma \longrightarrow 0
$$

and the map $m: \pi_{1}(\mathrm{M}) \rightarrow \Gamma=\mathrm{Hol} / \mathrm{Hol}^{0}$ is called the monodromy map. It can be interpreted geometrically as follows. The parallel translation along a contractible loop in $M$ always lies in $\mathrm{Hol}^{0}$ since it can be joined to the identity by the parallel translations along a family of paths which define a homotopy to the trivial loop. Thus, the parallel translation along any loop, regarded mod $\mathrm{Hol}^{0}$, only depends on the represented homotopy class, and this yields the monodromy map.

We finish this section by mentioning the following result.

Theorem 2.4 [46] Let $P \rightarrow M$ be a principal $G$ bundle, let $H \subset G$ be a (possibly non-regular) Lie subgroup. Moreover, let $P_{0} \subset P$ be a connected (possibly non-embedded) $H$-reduction of $P$. Then there is a connection on $P$ such that $P_{0}$ is the holonomy reduction of this connection.

In particular, there is a connection on $P$ with holonomy group $H$ if and only if $P$ admits a connected $H$-reduction $P_{0} \subset P$.

Proof. Any connection on $P_{0}$ can be extended to a connection on $P$ in a unique way, using the $G$-equivariance of the connection form. Thus, the problem reduces to showing that the principal $H$-bundle $P_{0} \rightarrow M$ has a connection whose holonomy equals all of $H$.

If we pick a "generic" (i.e., maximally non-integrable) horizontal distribution in the neighborhood of some $p \in P_{0}$, then $\left\{\Omega(v, w) \mid v, w \in \mathscr{H}_{p}\right\}=\mathfrak{h}$. Thus, by Ambrose-Singer holonomy Theorem 2.1, the holonomy reduction has the same dimension as $P_{0}$, and since $P_{0}$ is connected, it is the holonomy reduction, showing that $H$ is the holonomy group.

If $H \subset G$ is a regular subgroup, then the existence of an $H$-reduction is equivalent to the existence of a global section of the $G / H$-fiber bundle $P / H \rightarrow M$. That is, the existence of a connection with prescribed holonomy is merely a topological property.

\subsection{Connections on vector bundles}

Let $P \rightarrow M$ be a principal $G$-bundle, and let $\rho: G \rightarrow \operatorname{Aut}(V)$ be a representation on a finite dimensional (real or complex) vector space. Then the associated vector bundle is the bundle

$$
E:=P \times_{G} V \longrightarrow M,
$$

where $P \times{ }_{G} V$ is the quotient of $P \times V$ by the free $G$-action $g \star(p, v):=\left(p \cdot g^{-1}, \rho(g) v\right)$. Evidently, the fibers of $E$ are isomorphic to $V$. In fact, every vector bundle $E \rightarrow M$ can be described (nonuniquely) in this way: we fix a (real or complex) vector space $V$ isomorphic to the fibers of $E$, and let

$$
P_{E}:=\left\{u_{x}: E_{x} \rightarrow V \text { a linear isomorphism, where } x \in M\right\}
$$


with the obvious projection to $M$. This is called the full frame bundle of $E$. The structure group of $P_{E}$ is $\operatorname{Aut}(V)$ which acts by composition from the right, and it is straightforward to verify that $P_{E} \rightarrow M$ becomes a principal Aut $(V)$-bundle, and $E=P_{E} \times \operatorname{Aut}(V) V$ with the natural action of $\operatorname{Aut}(V)$ on $V$.

In general, if $E=P \times_{G} V$ is such a vector bundle and $\omega$ is a connection on $P$, then the splitting (1) of $T P$ induces a splitting

$$
T_{(p, v)}(P \times V)=\mathscr{H}_{p} \oplus \mathscr{V}_{p} \oplus V
$$

and since $\mathscr{H}$ is invariant under the diagonal action of $G$, it descends to a distribution $\mathscr{H}_{E} \subset T E$ on $E=P \times{ }_{G} V$, which is transversal to the fibers of $E \rightarrow M$. Since $\operatorname{ker}(d \pi)=E$ as a bundle in a canonical way, the connection $\omega$ on $P$ induces a bundle splitting

$$
T E=\mathscr{H}_{E} \oplus E
$$

Thus, we have an induced projection $T E \rightarrow E$, and this defines a covariant derivative on $E$, i.e., a map

$$
\nabla: \Gamma^{\infty}(M, E) \longrightarrow \Omega^{1}(M) \otimes \Gamma^{\infty}(M, E) \quad \text { as } \quad \nabla \sigma:=(d \sigma)_{E} .
$$

Let $\underline{c}:[a, b] \rightarrow M$ be a (piecewise smooth) path, pick a horizontal lift $c:[a, b] \rightarrow P$ and some $v_{0} \in V$. We let $v:[a, b] \rightarrow E$ be defined as $v(t):=\left(c(t), v_{0}\right) / G \in P \times_{G} V=E$. Then $v$ is parallel along $\underline{c}$, i.e., $\nabla_{\underline{c}^{\prime}(t)} v(t)=0$. Thus, as in the case of a connection on a principal bundle, we have the notion of parallel translation

$$
P_{\underline{c}}^{E}: E_{\underline{c}(a)} \longrightarrow E_{\underline{c}}(b)
$$

which is a linear isomorphism. Thus, the definition of the holonomy group of $\nabla$ is given analogously as

$$
\operatorname{Hol}_{x}(E \rightarrow M, \nabla):=\left\{P_{\underline{c}}^{E} \mid \underline{c}:[a, b] \rightarrow M \text { a path with } \underline{c}(a)=\underline{c}(b)=x\right\} \subset \operatorname{Aut}\left(E_{x}\right) .
$$

If $c:[a, b] \rightarrow P$ is a horizontal lift of some loop, then $c(b)=c(a) \cdot g$ for some $g \in G$, and hence, $v(b)=g \cdot v(a)$ with $v(t) \in E_{\underline{c}(t)}$ as above. Therefore, we have the following

Proposition 2.5 Let $P \rightarrow M$ be a principal $G$-bundle and let $E:=P \times_{G} V$ be an associated principal bundle w.r.t. some representation $\rho: G \rightarrow$ Aut $(V)$. Let $\omega \in \Omega^{1}(P) \otimes \mathfrak{g}$ be a connection on $P$ and let $\nabla: \Gamma^{\infty}(M, E) \longrightarrow \Omega^{1}(M) \otimes \Gamma^{\infty}(M, E)$ be the induced covariant derivative on $E$.

Then for $p \in P$ and $x:=\pi(p) \in M$ we have $\operatorname{Hol}_{x}(E \rightarrow M, \nabla) \cong \rho\left(\operatorname{Hol}_{p}\right) \subset A u t(V)$. In particular, if $P_{0} \subset P$ is the holonomy reduction of $\omega$, then $E=P_{0} \times_{H o l} V$.

Therefore, connections on vector bundles and their holonomies can be described in terms of the holonomy on an associated principle bundle.

\subsection{The Spencer complex}

We shall briefly summarize the construction of the Spencer complex for a Lie subalgebra $\mathfrak{g} \subset$ $\operatorname{End}(V)$. For a more detailed exposition, we refer the interested reader to $[18,44,58]$. 
Let $V$ be a finite dimensional vector space over $\mathbb{R}$ or $\mathbb{C}$. We let $A^{p, q}(V):=\odot^{p} V^{*} \otimes \Lambda^{q} V^{*}$. This space can be thought of as the space of $q$-forms on $V$ with values in the space of homogeneous polynomials on $V$ of degree $p$. Exterior differentiation thus yields a map $\delta: A^{p, q}(V) \rightarrow A^{p-1, q+1}(V)$, which turns $A^{*, *}(V)=\bigoplus_{p, q \geq 0} A^{p, q}(V)$ into a bigraded complex. Likewise, $\bigoplus_{p, q \geq 0}\left(V \otimes A^{p, q}(V)\right)$ becomes a bigraded complex by the maps $\delta_{V}:=I d_{V} \otimes \delta$.

Let $\mathfrak{g} \subset \operatorname{End}(V) \cong V^{*} \otimes V$ be a subalgebra. The $k$-th prolongation of $\mathfrak{g}$, denoted by $\mathfrak{g}^{(k)}$ for an integer $k$, is defined inductively by the formulae $\mathfrak{g}^{(-1)}=V, \mathfrak{g}^{(0)}=\mathfrak{g}$, and

$$
\mathfrak{g}^{(k)}=\delta_{V}^{-1}\left(\mathfrak{g}^{(k-1)} \otimes V^{*}\right)
$$

That is,

$$
\mathfrak{g}^{(k)}=\left(\mathfrak{g} \otimes \odot^{k} V^{*}\right) \cap\left(V \otimes \odot{ }^{k+1} V^{*}\right),
$$

where we use exterior differentiation $\delta: \odot{ }^{k+1} V^{*} \rightarrow V^{*} \otimes \odot^{k} V^{*}$ to regard both $\mathfrak{g} \otimes \odot^{k} V^{*}$ and $V \otimes$ $\odot^{k+1} V^{*}$ as subspaces of $V \otimes V^{*} \otimes \odot{ }^{k} V^{*}$. Alternatively, we can define $\mathfrak{g}^{(k)}$ inductively by $\mathfrak{g}^{(-1)}=V$, $\mathfrak{g}^{(0)}=\mathfrak{g}$ and the exact sequence

$$
0 \longrightarrow \mathfrak{g}^{(k)} \longrightarrow \mathfrak{g}^{(k-1)} \otimes V^{*} \longrightarrow \mathfrak{g}^{(k-2)} \otimes \Lambda^{2} V^{*} .
$$

For example,

$$
\mathfrak{g}^{(1)}=\left\{\alpha \in V^{*} \otimes \mathfrak{g} \mid \alpha(x) y=\alpha(y) x \text { for all } x, y \in V\right\} .
$$

Furthermore, we define the Spencer complex of $\mathfrak{g}$ to be $\left(C^{p, q}(\mathfrak{g}), \delta\right)$ with

$$
C^{p, q}(\mathfrak{g})=\mathfrak{g}^{(p-1)} \otimes \Lambda^{q}\left(V^{*}\right) \subset V \otimes \odot^{p} V^{*} \otimes \Lambda^{q} V^{*}=V \otimes A^{p, q}(V) .
$$

It is not hard to see that $\delta\left(C^{p, q}(\mathfrak{g})\right) \subset C^{p-1, q+1}(\mathfrak{g})$, and thus, $\left(C^{p, q}(\mathfrak{g}), \delta\right)$ is indeed a complex where we denote the boundary maps by

$$
\delta_{\mathfrak{g}}^{p, q}: C^{p, q}(\mathfrak{g}) \longrightarrow C^{p-1, q+1}(\mathfrak{g}) .
$$

Its cohomology groups $H^{p, q}(\mathfrak{g})$ are called the Spencer cohomology groups of $\mathfrak{g}$. The lower corner of this bigraded complex takes the form

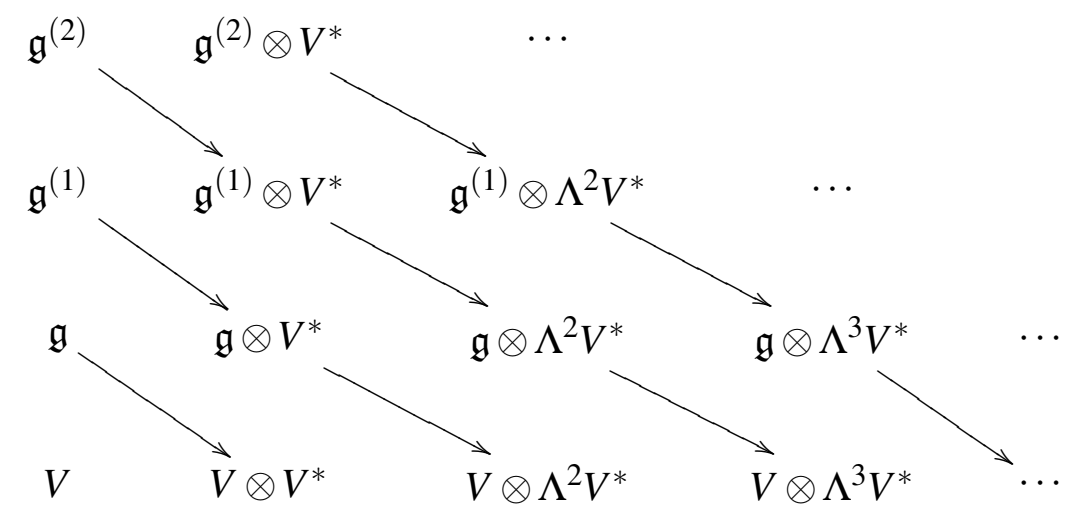


Table 4: List OF IRREDUCIBLE COMPLEX MATRIX LIE GROUPS G WITH $\mathfrak{g}^{(1)} \neq 0$

\begin{tabular}{|c|c|c|c|c|c|c|}
\hline & group G & \multicolumn{2}{|c|}{ representation $V$} & $\mathfrak{g}^{(1)}$ & $\mathfrak{g}^{(2)}$ & $H^{1,2}(\mathfrak{g})$ \\
\hline 1 & $\operatorname{SL}(n, \mathbb{C})$ & $\mathbb{C}^{n}$ & $n \geq 2$ & $\left(V \otimes \odot^{2} V^{*}\right)_{0}$ & $\left(V \otimes \odot^{3} V^{*}\right)_{0}$ & $\odot^{2} V^{*}$ \\
\hline 2 & $\operatorname{GL}(n, \mathbb{C})$ & $\mathbb{C}^{n}$ & $n \geq 1$ & $V \otimes \odot^{2} V^{*}$ & $V \otimes \odot^{3} V^{*}$ & 0 \\
\hline 3 & $\operatorname{GL}(n, \mathbb{C})$ & $\odot^{2} \mathbb{C}^{n}$ & $n \geq 2$ & $V^{*}$ & 0 & 0 \\
\hline 4 & $\operatorname{GL}(n, \mathbb{C})$ & $\Lambda^{2} \mathbb{C}^{n}$ & $n \geq 5$ & $V^{*}$ & 0 & 0 \\
\hline 5 & $\mathrm{GL}(m, \mathbb{C}) \cdot \mathrm{GL}(n, \mathbb{C})$ & $\mathbb{C}^{m} \otimes \mathbb{C}^{n}$ & $m, n \geq 2$ & $V^{*}$ & 0 & 0 \\
\hline 6 & $\operatorname{Sp}(n, \mathbb{C})$ & $\mathbb{C}^{2 n}$ & $n \geq 2$ & $\odot{ }^{3} V^{*}$ & $\odot{ }^{4} V^{*}$ & 0 \\
\hline 7 & $\mathbb{C}^{*} \cdot \operatorname{Sp}(n, \mathbb{C})$ & $\mathbb{C}^{2 n}$ & $n \geq 2$ & $\odot^{3} V^{*}$ & $\odot{ }^{4} V^{*}$ & 0 \\
\hline 8 & $\mathrm{CO}(n, \mathbb{C})$ & $\mathbb{C}^{n}$, & $n \geq 3$ & $V^{*}$ & 0 & $\mathscr{W}^{1}$ \\
\hline 9 & $\mathbb{C}^{*} \cdot \operatorname{Spin}(10, \mathbb{C})$ & $\mathbb{C}^{16}$ & & $V^{*}$ & 0 & 0 \\
\hline 10 & $\mathbb{C}^{*} \cdot \mathrm{E}_{6}^{\mathbb{C}}$ & $\mathbb{C}^{27}$ & & $V^{*}$ & 0 & 0 \\
\hline
\end{tabular}

It is worth pointing out that all of these spaces are $\mathfrak{g}$-modules in an obvious way, and that all maps are $\mathfrak{g}$-equivariant. Thus, the Spencer cohomology groups are $\mathfrak{g}$-modules as well. Also, we define $K(\mathfrak{g}):=\operatorname{ker} \delta_{\mathfrak{g}}^{1,2}$, so that we have the exact sequence

$$
0 \longrightarrow \mathfrak{g}^{(2)} \longrightarrow \mathfrak{g}^{(1)} \otimes V^{*} \longrightarrow K(\mathfrak{g}) \longrightarrow H^{1,2}(\mathfrak{g}) \longrightarrow 0,
$$

where the map in the middle is given by $R_{\alpha \otimes \phi}(x, y)=\phi(x) \alpha(y)-\phi(y) \alpha(x)$ for $\alpha \otimes \phi \in \mathfrak{g}^{(1)} \otimes V^{*}$.

If we assume that $\mathfrak{g} \subset \operatorname{End}(V)$ acts irreducibly, then there are only very few possibilities for which $\mathfrak{g}^{(1)} \neq 0$. These subalgebras have been classified by Cartan ([29]) and Kobayashi and Nagano ([53]). The result is listed in Table 4 for complex Lie algebras. The Spencer cohomologies $H^{1,2}(\mathfrak{g})$ of these Lie algebras are well-known. (See e.g. [18] and [56] who use considerably different techniques for the calculations).

\section{$2.4 G$-structures and intrinsic torsion}

Consider the tangent bundle $T M \rightarrow M$ of a connected manifold $M$. We define the (total) coframe bundle

$$
\mathfrak{F}_{V}:=\left\{u_{x}: T_{x} M \rightarrow V \text { a linear isomorphism }\right\} \longrightarrow M,
$$

where $\operatorname{dim} V=\operatorname{dim} M$ as in section 2.2. Thus, $\mathfrak{F}_{V} \rightarrow M$ is an $\operatorname{Aut}(V)$ - principal bundle. On $\mathfrak{F}_{V}$, we define the tautological one-form

$$
\theta \in \Omega^{1}\left(\mathfrak{F}_{V}\right) \otimes V, \quad \theta_{u}(v):=u(\mathrm{~d} \pi(v)) \text { for } v \in T_{u}\left(\mathfrak{F}_{V}\right),
$$

where $\pi: T M \rightarrow M$ denotes the canonical projection. We have the equivariance condition

$$
r_{g}^{*}(\theta)=g \cdot \theta
$$


Let $G \subset \operatorname{Aut}(V)$ be a (possibly non-regular) Lie subgroup. A $G$-structure on $M$ is, by definition, a reduction of $\mathfrak{F}_{V}$ with structure group $G$, i.e., it is a (possibly non-regular) submanifold $F \subset \mathfrak{F}_{V}$ such that we have the commuting diagram

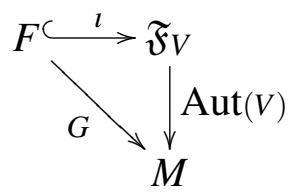

Note that if $G \subset \operatorname{Aut}(V)$ is a regular subgroup, then these reductions are in a one-to-one correspondence to sections of the $\operatorname{Aut}(V) / G$-bundle $\mathfrak{F}_{V} / G \rightarrow M$. Also, $\imath^{*}(\theta)$ is called the tautological one form of $F$, and we shall denote it by $\theta$ instead of $\imath^{*}(\theta)$. In fact, the existence of such a form $\theta \in \Omega^{1}(F) \otimes V$ characterizes $G$-structures on $M$ as the next result shows.

Proposition 2.6 Let $\pi: P \rightarrow M$ be a principal $G$-bundle and let $V$ be a vector space of the same dimension as $M$. If there exists one form $\underline{\theta} \in \Omega^{1}(P) \otimes V$ with $\operatorname{ker}(\underline{\theta})=\operatorname{ker}(d \pi)$ and a faithful representation $\rho: G \rightarrow A u t(V)$ such that $r_{g}^{*}(\underline{\theta})=\rho(g) \cdot \underline{\theta}$ for all $g \in G$, then there is a $G$-invariant immersion $\imath: P \hookrightarrow \mathfrak{F}_{V}$ such that

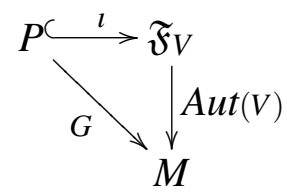

commutes and $\underline{\theta}=\iota^{*}(\theta)$, where $\theta$ is the tautological one form on $\mathfrak{F}_{V}$. In particular, $\imath(P) \subset \mathfrak{F}_{V}$ is a G-structure on $M$ with tautological form $\underline{\theta}$.

Proof. Since for $p \in P$ we have $\operatorname{kerd} \pi_{p}=\operatorname{ker} \underline{\theta}_{p}$, it follows that there is a unique isomorphism $\iota_{p}: T_{\pi(p)} M \rightarrow V$ such that $\underline{\theta}_{p}=\mathrm{d} \pi_{p} \circ \imath_{p}$. Thus, $\iota_{p} \in \mathfrak{F}_{V}$, so that we get a smooth map $\imath: P \rightarrow \mathfrak{F}_{V}$. The equivariance of $\underline{\theta}$ implies that $\imath$ is $G$-equivariant, hence $\imath(P) \subset \mathfrak{F}_{V}$ is a $G$-structure, and the fact that $\underline{\theta}=\imath^{*}(\theta)$ follows immediately from definition (15).

Note that $\operatorname{End}(V)$ is the Lie algebra of $\operatorname{Aut}(V)$, hence any connection on $\mathfrak{F}_{V}$ is a one form $\omega \in \Omega^{1}\left(\mathfrak{F}_{V}\right) \otimes \operatorname{End}(V)$. Its torsion is defined as

$$
\Theta:=\mathrm{d} \theta+\omega \wedge \theta \in \Omega^{2}\left(\mathfrak{F}_{V}\right) \otimes V
$$

whose derivative yields

$$
\Omega \wedge \theta=\mathrm{d} \Theta+\omega \wedge \Theta
$$

Then (17) implies the conditions

$$
\left.\xi^{*}\right\lrcorner \Theta=0 \text { for all } \xi \in \mathfrak{g} \text {, and } \mathrm{d} r_{g}^{*}(\Theta)=g \cdot \Theta \text {. }
$$

Therefore, there is a $\operatorname{Aut}(V)$-equivariant map Tor $: \mathfrak{F}_{V} \rightarrow \operatorname{Hom}\left(\Lambda^{2} V, V\right)$ such that

$$
\Theta=\operatorname{Tor}(\theta \wedge \theta)
$$


The equivariance of Tor implies that its derivative takes the form

$$
\mathrm{d} \text { Tor }+\omega \cdot \text { Tor }=\nabla_{\theta} \text { Tor }
$$

where the multiplication on the left hand side refers to the action of $\mathfrak{g} \subset \operatorname{End}(V)$ on $\Lambda^{2} V^{*} \otimes V$, and where $\nabla_{\theta}$ Tor $\in \Omega^{1}\left(\mathfrak{F}_{V}\right) \otimes\left(V^{*} \otimes \Lambda^{2} V^{*} \otimes V\right)$. Analogously, by (4), it follows that there is a $\operatorname{Aut}(V)$-equivariant map $R: \mathfrak{F}_{V} \rightarrow \operatorname{Hom}\left(\Lambda^{2} V, \operatorname{End}(V)\right)$ such that

$$
\Omega=R(\theta \wedge \theta)
$$

and (18) implies the first Bianchi identity

$$
\sum_{\text {cycl. }} R\left(v_{1}, v_{2}\right) v_{3}=\sum_{\text {cycl. }}\left(\nabla_{v_{1}} \operatorname{Tor}\right)\left(v_{2}, v_{3}\right)+\operatorname{Tor}\left(\operatorname{Tor}\left(v_{1}, v_{2}\right), v_{3}\right) .
$$

Again, by the $\operatorname{Aut}(V)$-equivariance of $R$, taking the derivative of (20) yields

$$
\mathrm{d} R+\omega \cdot R=\nabla_{\theta} R
$$

where the multiplication on the left hand side refers to the action of $\mathfrak{g} \subset \operatorname{End}(V)$ on $\Lambda^{2} V^{2} \otimes \operatorname{End}(V)$, and where $\nabla_{\theta} R \in \Omega^{1}\left(\mathfrak{F}_{V}\right) \otimes\left(V^{*} \otimes \Lambda^{2} V^{*} \otimes \operatorname{End}(V)\right)$. In fact, (3) implies that for all $v_{1}, v_{2}, v_{3} \in V$ we have the second Bianchi identity

$$
\sum_{\text {cycl. }}\left(\nabla_{v_{1}} R\right)\left(v_{2}, v_{3}\right)+R\left(\operatorname{Tor}\left(v_{1}, v_{2}\right), v_{3}\right)=0
$$

As $\operatorname{Hom}\left(\Lambda^{2} T M, T M\right)=\mathfrak{F}_{V} \times_{\operatorname{Aut}(V)} \operatorname{Hom}\left(\Lambda^{2} V, V\right)$ and $\operatorname{Hom}\left(\Lambda^{2} T M, \operatorname{End}(T M)\right)=\mathfrak{F}_{V} \times \operatorname{Aut}(V)$ $\operatorname{Hom}\left(\Lambda^{2} V, \operatorname{End}(V)\right)$ the equivarianve of Tor and $R$ implies that they induce sections which by abuse of notation we denote by the same symbols, namely

$$
\text { Tor } \in \Gamma^{\infty}\left(\operatorname{Hom}\left(\Lambda^{2} T M, T M\right)\right) \text { and } R \in \Gamma^{\infty}\left(\operatorname{Hom}\left(\Lambda^{2} T M, \operatorname{End}(T M)\right),\right.
$$

These sections are also called the torsion and the curvature of the connection, respectively. In terms of the covariant derivative on $T M$ corresponding to $\omega$ they are given by the formulas

$$
\operatorname{Tor}(X, Y)=\nabla_{X} Y-\nabla_{Y} X-[X, Y]
$$

and

$$
R(X, Y) Z=\nabla_{X} \nabla_{Y} Z-\nabla_{Y} \nabla_{X} Z-\nabla_{[X, Y]} Z \text {. }
$$

If $\omega_{F}$ is a connection one form on the $G$-bundle $F \rightarrow M$, then by $\operatorname{Aut}(V)$-equivariant continuation, there is a unique connection one form $\omega \in \Omega^{1}\left(\mathfrak{F}_{V}\right) \otimes \operatorname{End}(V)$ such that $\omega_{F}=\imath^{*}(\omega)$. The converse is not true; in fact, given a connection one form $\omega \in \Omega^{1}\left(\mathfrak{F}_{V}\right) \otimes \operatorname{End}(V)$, its restriction $\imath^{*}(\omega)$ is a connection one form on $F$ if and only if $F$ contains a holonomy reduction of $\omega$.

Let us now consider two connections $\omega_{F}$ and $\omega_{F}^{\prime}$ on a $G$ structure $F \hookrightarrow \mathfrak{F}_{V}$. By definition, $\omega_{F}\left(\xi^{*}\right)=\omega_{F}^{\prime}\left(\xi^{*}\right)=\xi$ for all $\xi \in \mathfrak{g}$, hence there is a $G$ - equivariant map $\alpha: F \rightarrow \operatorname{Hom}(V, \mathfrak{g})$ such that

$$
\omega_{F}^{\prime}=\omega_{F}+\alpha \circ \theta
$$


This means that for the torsion two forms of $\omega_{F}$ and $\omega_{F}^{\prime}$ we have

$$
\Theta_{F}^{\prime}-\Theta_{F}=(\alpha \circ \theta) \wedge \theta=\delta_{\mathfrak{g}}^{1,1}(\alpha)(\theta \wedge \theta)
$$

with the map $\delta_{\mathfrak{g}}^{1,1}: \mathfrak{g} \otimes V^{*} \rightarrow V \otimes \Lambda^{2} V$ from the Spencer complex (13). That is,

$$
\operatorname{Tor}_{F}^{\prime}=\operatorname{Tor}_{F}+\delta_{\mathfrak{g}}^{1,1}(\alpha)
$$

This immediately implies the following

Theorem 2.7 Let $F \hookrightarrow \mathfrak{F}_{V}$ be a G-structure for some (possibly non-regular) Lie subgroup $G \subset$ Aut $(V)$ with Lie algebra $\mathfrak{g} \subset$ End $(V)$. Then the following hold.

1. Let $\omega_{F}$ be a connection on $F$, let Tor $: F \rightarrow \Lambda^{2} V^{*} \otimes V$ be its torsion and $[$ Tor $]: F \rightarrow H^{1,1}(\mathfrak{g})$ be the element represented by Tor in the Spencer cohomology group. Then [Tor] is independent of the choice of connection on $F$, and is thus called the intrinsic torsion of $F$.

2. There exists a torsion free connection on $F$, i.e., a connection with $\Theta \equiv 0$, if and only if the intrinsic torsion of $F$ vanishes.

3. If $\mathfrak{g}^{(1)}=0$, then the torsion of a connection on $F$ uniquely determines the connection.

Let us now assume that $\omega$ is a torsion free connection on the $G$-structure $F \hookrightarrow \mathfrak{F}_{V}$ on $M$. Then from (21) it follows that $\sum_{c y c l} R\left(v_{1}, v_{2}\right) v_{3}=0$ which means that the image of the curvature map $R: \mathfrak{F}_{V} \rightarrow \operatorname{Hom}\left(\Lambda^{2} V, \operatorname{End}(V)\right)$ is contained in $K(\mathfrak{g})$, the kernel of the map $\delta_{\mathfrak{g}}^{1,2}$ of the Spencer complex as defined in (14). This kernel may be describes as

$$
\begin{aligned}
K(\mathfrak{g}) & =\operatorname{ker} \delta_{\mathfrak{g}}^{1,2}=\operatorname{Hom}\left(\Lambda^{2} V, \mathfrak{g}\right) \cap\left(\operatorname{ker} \delta^{1,2}\right) \\
& =\left\{R \in \operatorname{Hom}\left(\Lambda^{2} V, \mathfrak{g}\right) \mid \sum_{\text {cycl. }} R\left(v_{1}, v_{2}\right) v_{3}=0\right\}
\end{aligned}
$$

and is called the space of formal curvatures of $\mathfrak{g} \subset \operatorname{End}(V)$. Furthermore, (23) implies that for a torsion free connection we have

$$
\begin{aligned}
\nabla R: F \longrightarrow K^{1}(\mathfrak{g}): & \operatorname{Hom}(V, K(\mathfrak{g})) \cap \operatorname{ker}\left(V^{*} \otimes \Lambda^{2} V^{*} \otimes \mathfrak{g} \longrightarrow \Lambda^{3} V^{*} \otimes \mathfrak{g}\right) \\
& =\left\{\phi \in \operatorname{Hom}(V, K(\mathfrak{g})) \mid \sum_{\text {cycl. }} \phi\left(v_{1}\right)\left(v_{2}, v_{3}\right)=0\right\} .
\end{aligned}
$$

These two identities for torsion free connections have some remarkable consequences. For a Lie subalgebra $\mathfrak{g} \subset \operatorname{End}(V)$ we define the ideal

$$
\underline{\mathfrak{g}}:=\{R(v, w) \mid R \in K(\mathfrak{g}), v, w \in V\} \triangleleft \mathfrak{g}
$$

If $\omega \in \Omega^{1}(P) \otimes \mathfrak{g}$ is a torsion free connection on some $G$-structure $P \hookrightarrow \mathfrak{F}_{V}$ on some manifold $M$, then the linear maps

$$
\Lambda^{2} T_{p} P \longrightarrow \mathfrak{h o l}_{p}, \quad v \wedge w \longmapsto \Omega\left(\mathrm{d}_{\underline{c}}(v), \mathrm{d} \Pi_{\underline{c}}(w)\right)
$$


where $\underline{c}:[a, b] \rightarrow M$ is any path with $\underline{c}(a)=\pi(p)$, satisfy the first Bianchi identity and hence are elements of $K\left(\mathfrak{h o l}_{p}\right)$. Therefore, $\Omega\left(\mathrm{d}_{\underline{c}}(v), \mathrm{d}_{\underline{c}}(w)\right) \in \underline{h o l}_{p} \triangleleft \mathfrak{h o l} \mathfrak{l}_{p}$. On the other hand, the AmbroseSinger Holonomy Theorem 2.1 immediately implies that $\mathfrak{h o l}_{p}$ is generated by $\Omega\left(\mathrm{d} \Pi_{\underline{c}}(v), \mathrm{d} \Pi_{\underline{c}}(w)\right)$, so that we must have

$$
\underline{\mathfrak{h o l}} p=\mathfrak{h o l}_{p} \text {. }
$$

Furthermore, if $K^{1}(\mathfrak{g})=0$, then evidently $\nabla R \equiv 0$, and a torsion free connection with this property is called locally symmetric. Thus, we can deduce the following

Theorem 2.8 (Berger's criteria [9]) Let $\mathfrak{g} \subset \operatorname{End}(V)$ be a Lie subalgebra, and define $K(\mathfrak{g}), K^{1}(\mathfrak{g})$ and $\mathfrak{g} \triangleleft \mathfrak{g}$ as above.

1. If $\mathfrak{g}$ is the Lie algebra of the holonomy group of a torsion free connection on some manifold, then $\underline{\mathfrak{g}}=\mathfrak{g}$.

2. If $K^{1}(\mathfrak{g})=0$, then any torsion free connection on some manifold whose holonomy Lie algebra is contained in $\mathfrak{g}$ must be locally symmetric.

Lie algebras $\mathfrak{g} \subset \operatorname{End}(V)$ satisfying $\mathfrak{g}=\mathfrak{g}$ are called Berger subalgebras. Moreover, a Berger algebra is called symmetric if $K^{1}(\mathfrak{g})=0$ and non-symmetric otherwise. Thus, Theorem 2.8 says that the Lie algebra of the holonomy group of a torsion free connection must be a Berger algebra, and if this Berger algebra is symmetric, then any torsion free connection with this holonomy must be locally symmetric.

We shall describe locally symmetric connections in more detail in the following section.

\subsection{Symmetric connections}

Let $H \subset G$ be a closed Lie subgroup with Lie algebra $\mathfrak{h} \subset \mathfrak{g}$, so that $M:=G / H$ is a homogeneous space. Furthermore, assume that the Lie algebra $\mathfrak{g}$ of $G$ admits an $A d_{H^{-}}$invariant decomposition

$$
\mathfrak{g}=\mathfrak{h} \oplus \mathfrak{m} .
$$

The $A d_{H}$-invariance implies that also

$$
[\mathfrak{h}, \mathfrak{m}] \subset \mathfrak{m}
$$

Let $\mu \in \Omega^{1}(G) \otimes \mathfrak{g}$ be the left invariant Maurer-Cartan form of $G$ and decompose it according to (24) as

$$
\mu=\omega+\theta, \text { where } \omega \in \Omega^{1}(G) \otimes \mathfrak{h} \text { and } \theta \in \Omega^{1}(G) \otimes \mathfrak{m} .
$$

Since $\mu$ is left invariant, it satisfies the equivariance condition $r_{g^{-1}} *(\mu)=A d_{g} \circ \mu$, hence the corresponding equivariance holds for $\omega$ and $\theta$ as well. Moreover, the action fields $\xi^{*}$ of the right $H$-action on $G$ are left invariant, hence $\omega\left(\xi^{*}\right)=\mu\left(\xi^{*}\right)=\xi$ for all $\xi \in \mathfrak{h}$. It follows that $\omega$ is a connection one form on $G \rightarrow M$, and by Proposition 2.6, we may regard $G$ as an $H$-structure on $M$ and $\theta$ as the tautological form of this $H$-structure. The Maurer-Cartan equation

$$
\mathrm{d} \mu+\frac{1}{2}[\mu, \mu]=0
$$


implies for the torsion and the curvature of this connection

$$
\Theta=-\frac{1}{2}[\theta, \theta]_{\mathfrak{m}} \text { and } \Omega=-\frac{1}{2}[\theta, \theta]_{\mathfrak{h}} .
$$

Thus, $\omega$ is torsion free if and only if $[\mathfrak{m}, \mathfrak{m}] \subset \mathfrak{h}$ which together with (25) implies that the involution

$$
\mathrm{d} \sigma: \mathfrak{g} \longrightarrow \mathfrak{g},\left.\quad \mathrm{d} \sigma\right|_{\mathfrak{h}}=I d_{\mathfrak{h}},\left.\quad \mathrm{d} \sigma\right|_{\mathfrak{m}}=-I d_{\mathfrak{m}}
$$

is a Lie algebra isomorphism and hence - after passing to an appropriate covering of $G$ - is the differential of a Lie group involution

$$
\sigma: G \rightarrow G, \quad \sigma^{2}=I d_{G}
$$

This process can be reverted, and we obtain the following result.

Proposition 2.9 Let $\sigma: G \rightarrow G$ be an involution, i.e., an isomorphism with $\sigma^{2}=I d_{G}$, and let $\mathfrak{g}=\mathfrak{h} \oplus \mathfrak{m}$ be the eigenspace decomposition of $\mathfrak{g}$ w.r.t. the differential $d \sigma: \mathfrak{g} \rightarrow \mathfrak{g}$. Let $H \subset G$ be any $\sigma$-invariant subgroup with Lie algebra $\mathfrak{h}$. Then the following hold.

1. The principal $H$-bundle $G \rightarrow G / H=: M$ is an $H$-structure on $M$ and carries a canonical $G$ invariant torsion free connection induced by the splitting $\mathfrak{g}=\mathfrak{h} \oplus \mathfrak{m}$ from (24).

2. For each $p \in G / H$, there is a connection preserving involution $\sigma_{p}: G / H \rightarrow G / H$ such that $p$ is an isolated fixed point of $\sigma_{p}$.

Thus, $G / H$ is an (affine) symmetric space. Moreover, the Lie algebra of the holonomy group of the canonical connection is $[\mathfrak{m}, \mathfrak{m}] \triangleleft \mathfrak{h}$.

Indeed, for $p=g H$ and $q=g^{\prime} H \in G / H$, the involution $\sigma_{p}$ is defined as

$$
\sigma_{p}(q):=g \sigma\left(g^{\prime}\right) g^{-1} H .
$$

The statement on the holonomy algebra follows once again from the Ambrose-Singer-Holonomy Theorem 2.1.

From (22) and the definition of the symmetric connection it follows immediately that $\nabla R \equiv 0$. In fact, this equation characterizes symmetric connections, at least locally.

Proposition 2.10 Let $P \rightarrow M$ be an $H$-structure with a torsion free connection $\omega$ such that $\nabla R \equiv 0$. Then the connection is locally symmetric, i.e., after replacing $M, P$ and $H$ by appropriate covers, there are local diffeomorphism $\imath: P \rightarrow G$ and $\underline{\imath}: M \rightarrow G / H$, where $G$ is a Lie group containing $H$ such that $G / H$ is a symmetric space, and so that the diagram

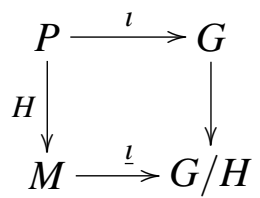

commutes. Moreover, if $\mu=\theta_{\mathfrak{g}}+\omega_{\mathfrak{g}}$ is the decomposition of the Maurer-Cartan form of $G$ which induces the symmetric connection, then $\theta=\imath^{*}\left(\theta_{\mathfrak{g}}\right)$ and $\omega=\imath^{*}\left(\omega_{\mathfrak{g}}\right)$. That is, $\underline{\imath}: M \rightarrow G / H$ is a connection preserving local diffeomorphism. 
Proof. We define a Lie algebra structure on $\mathfrak{g}:=\mathfrak{h} \oplus V$ by the conditions that

1. $\mathfrak{h} \subset \mathfrak{g}$ is a Lie subalgebra.

2. $[\mathfrak{h}, V] \subset V$, and $\left.a d_{h}\right|_{V} \in \operatorname{End}(V)$ is given by the embedding $\mathfrak{h} \subset \operatorname{End}(V)$ for $h \in \mathfrak{h}$.

3. $[V, V] \subset \mathfrak{h}$ is given by requiring the identity $\omega\left(v_{1}, v_{2}\right)=-\left[\theta\left(v_{1}\right), \boldsymbol{\theta}\left(v_{2}\right)\right]$ for all $v_{1}, v_{2} \in T_{p} P$.

It is straightforward to verify that the condition $\nabla R \equiv 0$ implies that the definition of $[V, V]$ is independent of the choice of $p \in P$, and that this is indeed a Lie algebra structure on $\mathfrak{g}=\mathfrak{h} \oplus V$.

Thus, we may define the form $\mu:=\omega+\theta \in \Omega^{1}(P) \otimes \mathfrak{g}$, and the structure equations (2) and (17) with $\Theta=0$ imply that $\mu$ satisfies the Maurer- Cartan equation (26). Therefore, by Cartan's theorem, after replacing $P$ by an appropriate cover, there is a local diffeomorphism $\imath: P \rightarrow G$ with $\mu=\imath^{*}\left(\mu_{G}\right)$, where $G$ is a Lie group with Lie algebra $\mathfrak{g}$, and $\mu_{G}$ is the Maurer-Cartan form on $G$.

The involution $\mathrm{d} \sigma: \mathfrak{g} \rightarrow \mathfrak{g}$ which has $\mathfrak{h}$ and $V$ as its $(+1)$ and (-1)-eigenspace, respectively, integrates to an involution $\sigma: G \rightarrow G$, and since we assume $H$ to be connected, it follows that $G / \imath(H)$ is a symmetric space, and after identifying $H$ and $\iota(H)$, it follows that $\iota: P \rightarrow G$ induces a connection preserving map $\underline{\underline{\imath}}: M \rightarrow G / H$.

\subsection{Splitting theorems}

Let $P_{i} \rightarrow M_{i}$ be principal $G_{i}$-bundles for $i=1,2$. The product bundle $P \rightarrow M$ is the principal $\left(G_{1} \times G_{2}\right)$-bundle $P:=P_{1} \times P_{2}$ with $M:=M_{1} \times M_{2}$.

If $\omega_{i} \in \Omega^{1}\left(P_{i}\right) \otimes \mathfrak{g}_{i}$ is a connection on $P_{i}$, then $\omega:=p_{1}^{*}\left(\omega_{1}\right)+p_{2}^{*}\left(\omega_{2}\right) \in \Omega^{1}(P) \otimes\left(\mathfrak{g}_{1} \oplus \mathfrak{g}_{2}\right)$ with the canonical projections $p_{i}: P \rightarrow P_{i}$ is again a connection one form on $P$ and is called the product connection of $\omega_{1}$ and $\omega_{2}$ on $P$. It follows that the curvature $\Omega \in \Omega^{2}(P) \otimes\left(\mathfrak{g}_{1} \oplus \mathfrak{g}_{2}\right)$ of the product connection is given by

$$
\Omega=p_{1}^{*}\left(\Omega_{1}\right)+p_{2}^{*}\left(\Omega_{2}\right) .
$$

For the equivalence relation $\sim$ from $(8)$ it now follows from the definition that

$$
\left(p_{1}, p_{2}\right) \sim\left(q_{1}, q_{2}\right) \text { in } P \text { if and only if } p_{i} \sim q_{i} \text { in } P_{i} \text { for } i=1,2 .
$$

Thus, since by the proof of the Ambrose-Singer Holonomy Theorem 2.1 the holonomy reduction of $P \rightarrow M$ is a single equivalence class w.r.t. $\sim$, it follows that a holonomy reduction $P_{0} \subset P$ is of the form $P_{0}=P_{0}^{1} \times P_{0}^{2}$, where $P_{0}^{i} \subset P_{i}$ are holonomy reductions. In particular, for the holonomy group we have

$$
\operatorname{Hol}_{\left(p_{1}, p_{2}\right)}(P)=\operatorname{Hol}_{p_{1}}\left(P_{1}\right) \times \operatorname{Hol}_{p_{2}}\left(P_{2}\right) \subset G_{1} \times G_{2} .
$$

Similarly, if $P_{i} \hookrightarrow \mathfrak{F}_{V_{i}}$ is a $G_{i}$-structure on $M_{i}$ and $\theta_{i} \in \Omega^{1}\left(P_{i}\right) \otimes V_{i}$ is the corresponding tautological form for $i=1,2$, then $\theta:=p_{1}^{*}\left(\theta_{1}\right)+p_{2}^{*}\left(\theta_{2}\right) \in \Omega^{1}(P) \otimes\left(V_{1} \oplus V_{2}\right)$ satisfies the hypotheses of Proposition 2.6, hence there is an induced $\left(G_{1} \times G_{2}\right)$-structure $P \hookrightarrow \mathfrak{F}_{V_{1}} \oplus V_{2}$ on $M$. This structure is called the product structure of $P_{1} \hookrightarrow \mathfrak{F}_{V_{1}}$ and $P_{2} \hookrightarrow \mathfrak{F}_{V_{2}}$. 
In the following, we shall derive some conditions which imply that a connection is a product connection. For this, let us consider a $G$-structure $P \subset \mathfrak{F}_{V} \rightarrow M$. If there is a $G$-invariant decomposition

$$
V=V_{1} \oplus \ldots \oplus V_{r}
$$

with $r \geq 2$ and $V_{j} \neq 0$ for all $j$, then we call $G \subset \operatorname{Aut}(V)$ decomposable, otherwise, $G \subset \operatorname{Aut}(V)$ is called indecomposable.

Theorem 2.11 Let $M$ be a manifold with a torsion free connection, and suppose that the holonomy

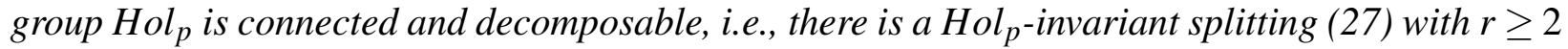
and $V_{j} \neq 0$ and $V \cong T_{p} M$. Let $H_{i} \subset$ Aut $\left(V_{i}\right), i=1, \ldots, r$, be the restriction of the action on Hol ${ }_{p}$ on $V_{i}$, and let $\mathfrak{h}_{i} \subset \operatorname{End}\left(V_{i}\right)$ be its Lie algebra.

Suppose further that the first prolongations of $\mathfrak{h}_{i}$ vanish, i.e., $\mathfrak{h}_{i}^{(1)}=0$ for $i=1, \ldots, r$. Then the following hold.

1. The connection is locally a product connection, i.e., each $x \in M$ has a neighborhood $U=$ $U_{1} \times \ldots \times U_{r}$ such that the restriction of the connection to $U \subset M$ is a product of connections on $U_{i}$.

2. $\mathrm{Hol}_{p}=H_{1} \times \ldots \times H_{r}$, where $H_{i}$ acts trivially on $V_{j}$ for $i \neq j$.

Proof. Let $\pi: P \rightarrow M$ be a holonomy reduction and let $\mathscr{H}$ be the horizontal distribution on $P$ which we decompose as

$$
\mathscr{H}=\mathscr{H}_{1} \oplus \ldots \oplus \mathscr{H}_{r}, \text { where } \mathscr{H}_{i}:=\mathscr{H} \cap \theta^{-1}\left(V_{i}\right) .
$$

The $G$-equivariance implies that there are distributions $\mathscr{D}_{i} \subset T M$ such that

$$
T M=\mathscr{D}_{1} \oplus \ldots \oplus \mathscr{D}_{r}, \text { and } \mathrm{d} \pi: \mathscr{H}_{i} \rightarrow \mathscr{D}_{i} \text { is a pointwise linear isomorphism. }
$$

We assert that for the space of formal curvatures, we have

$$
K\left(\mathfrak{h}_{1} \oplus \cdots \oplus \mathfrak{h}_{r}\right)=K\left(\mathfrak{h}_{1}\right) \oplus \ldots \oplus K\left(\mathfrak{h}_{r}\right) .
$$

The inclusion $\supset$ is evident. For the converse, consider an element of $R \in K\left(\mathfrak{h}_{1} \oplus \cdots \oplus \mathfrak{h}_{r}\right)$. Since $\left.R\left(V_{i}, V_{i}\right)\right|_{V_{i}} \in K\left(\mathfrak{h}_{i}\right)$, we may assume w.l.o.g. that $\left.R\left(V_{i}, V_{i}\right)\right|_{V_{i}}=0$. Then the first Bianchi identity for $R$ implies

$$
0=\underbrace{R\left(x_{i}, y_{j}\right) z_{k}}_{\in V_{k}}+\underbrace{R\left(y_{j}, z_{k}\right) x_{i}}_{\in V_{i}}+\underbrace{R\left(z_{k}, x_{i}\right) y_{j}}_{\in V_{j}},
$$

where the subscripts refer to the decomposition (27). Thus, if $i, j, k$ are pairwise different, then all terms vanish, so that we have $R\left(V_{i}, V_{j}\right) V_{k}=0$ in this case. Next, if $i=j \neq k$, then

$$
R\left(x_{i}, y_{i}\right) z_{k}=0 \text { and } R\left(z_{k}, y_{i}\right) x_{i}-R\left(z_{k}, x_{i}\right) y_{i}=0 .
$$

The first equation in (29) implies that $R\left(V_{i}, V_{i}\right) V_{k}=0$ for $k \neq i$, and therefore, $R\left(V_{i}, V_{i}\right)=0$. The second equation in (29) implies that for fixed $z_{k} \in V_{k}$, the map $V_{i} \rightarrow \mathfrak{h}_{i},\left.x_{i} \mapsto R\left(z_{k}, x_{i}\right)\right|_{V_{i}}$ is an 
element of $\mathfrak{h}_{i}^{(1)}$. Since by hypothesis $\mathfrak{h}_{i}^{(1)}=0$, it follows that $\left.R\left(z_{k}, x_{i}\right)\right|_{V_{i}}=0$. Thus, $R\left(V_{k}, V_{i}\right)=0$ for $k \neq i$ and hence, $R=0$ which shows (28).

This together with Theorem 2.8 implies that $\mathfrak{h o l}_{p} \subset \mathfrak{h}_{1} \oplus \ldots \oplus \mathfrak{h}_{r}$, and since the revers inclusion is obvious, we have equality. Thus, we also have the asserted equality for the holonomy groups as we assume these to be connected.

Let us decompose $\theta:=\theta_{1}+\ldots+\theta_{r}, \omega=\omega_{1}+\ldots+\omega_{r}$ and $\Omega=\Omega_{1}+\ldots+\Omega_{r}$ with $\theta_{i} \in$ $\Omega^{1}(P) \otimes V_{i}$ and $\omega_{i}, \Omega_{i} \in \Omega^{*}(P) \otimes \mathfrak{h}_{i}$, and we define the distributions

$$
\hat{\mathscr{H}}_{i}:=\mathscr{H}_{i} \oplus\left\{\xi^{*} \mid \xi \in \mathfrak{h}_{i}\right\} .
$$

Then $\left.\left.\hat{\mathscr{H}}_{i}\right\lrcorner \theta_{j}=0, \hat{\mathscr{H}}_{i}\right\lrcorner \omega_{j}=0$ and $\left.\hat{\mathscr{H}}_{i}\right\lrcorner \Omega_{j}=0$ for $i \neq j$. Thus $\hat{\mathscr{H}}_{i}$ is integrable, and

$$
\mathrm{d} \theta_{i}+\omega_{i} \wedge \theta_{i}=0 \text { and } \Omega_{i}=\mathrm{d} \omega_{i}+\frac{1}{2}\left[\omega_{i}, \omega_{i}\right] \text { for all } i .
$$

If we let $P_{i} \subset P$ be an integral leaf of $\hat{\mathscr{H}}_{i}$, then $U_{i}:=\pi\left(P_{i}\right) \subset M$ is an integral leaf of $\mathscr{D}_{i} \subset T M$, and the restriction $\pi: P_{i} \rightarrow U_{i}$ is a principal $H_{i}$ - bundle. Indeed, Proposition 2.6 implies that $\theta_{i}$ may be regarded as the tautological form of an $H_{i}$-structure $P_{i} \hookrightarrow \mathfrak{F}_{V_{i}}$ on $U_{i}$, and $\omega_{i}$ is a torsion free connection with holonomy $H_{i}$ on $P_{i}$.

Thus, each $x \in M$ has a neighborhood $U=U_{1} \times \ldots \times U_{r}$ where the $U_{i}$ are integral leafs of $\mathscr{D}_{i}$, and $\theta$ induces the product structure on $U$ with structure group $H_{1} \times \ldots \times H_{r}$, and $\omega$ is the product connection of the $\omega_{i}$ as asserted.

We would like to point out that in general, the decomposability of the holonomy group alone does not imply connection to be a direct sum connection in general. See [54, p.290] for an example. That is, the condition $\mathfrak{h}_{i}^{(1)}=0$ is essential in our argument.

As a special application of the splitting theorem, note that the prolongations of the orthogonal Lie algebras vanish, i.e., $\mathfrak{s o}(p, q)^{(1)}=0$. Thus, Theorem 2.11 implies the following results.

Theorem 2.12 (de Rham-Wu Splitting Theorem [37, 70]) Let $(M, g)$ be a (pseudo-)Riemannian manifold, and suppose that the holonomy group of its Levi-Civita connection is decomposable. Then locally, $(M, g)$ is isometric to a product metric $\left(\mathbb{R}^{k_{1}}, g_{1}\right) \times \ldots \times\left(\mathbb{R}^{k_{r}}, g_{r}\right)$ with $k_{j}=\operatorname{dim} V_{j}$, and $\operatorname{Hol}_{p}^{0}(M)=H_{1} \times \ldots \times H_{r}$ with $H_{j} \subset O\left(V_{j}, g_{j}\right)$.

This was first shown by de Rham in the Riemannian case and later generalized by Wu to the pseudo-Riemannian case. Note that in the Riemannian case, decomposability and irreducibility are equivalent.

In fact, there is also a global version of these splitting theorems which we shall not prove here. It relies on the Cartan-Ambrose-Hicks-theorem which explains the behavior of the curvature tensor under parallel translation. For a proof, see e.g. [4].

Theorem 2.13 (Global de Rham-Wu Splitting Theorem [37, 71]) Let $(M, g)$ be a geodesically complete simply connected (pseudo-)Riemannian manifold, and suppose that the holonomy group of its Levi-Civita connection is decomposable. Then $(M, g)=\left(M_{1}, g_{1}\right) \times \ldots \times\left(M_{r}, g_{r}\right)$ is the Riemannian product of complete (pseudo-)Riemannian manifolds $\left(M_{i}, g_{i}\right)$.

By virtue of this theorem, it is generally natural to assume the holonomy to be indecomposable as we may regard (local) product connections as "trivial" compositions. 


\section{Classification results}

In this section, we address the question which subgroups $G \subset \operatorname{Aut}(V)$ can occur as the holonomy of a given principal bundle. By Theorem 2.4, this reduces to deciding for which subgroups of the structure group there is a reduction. In particular, locally any connected subgroup $G \subset \operatorname{Aut}(V)$ can be realized as a holonomy group.

However, if we deal with torsion free connections on some $G$-structure, then the answer is far more difficult. By Theorem 2.8, for a subgroup $G \subset \operatorname{Aut}(V)$ to be the holonomy group of a torsion free connection, it is necessary that its Lie algebra is a Berger algebra. Therefore, the problem of classifying all holonomy groups of torsion free connections has as an algebraic subproblem the classification of all Berger algebras $\mathfrak{g} \subset \operatorname{End}(V)$.

Surprisingly, to the authors knowledge, there is no instance known of a Berger algebra which cannot be the Lie algebra of such a holonomy group. However, since there is no complete classification of Berger algebras, it is not clear if this is the case in general.

We shall now collect some classification results for certain subclasses of holonomy groups and algebras.

\subsection{Irreducible Symmetric spaces}

Recall from section 2.5 that a symmetric space is a manifold with an affine connection $(M, \nabla)$ such that for each $x \in M$ there is a connection preserving involution $\sigma_{x}: M \rightarrow M$ which has $x \in M$ as an isolated fixed point. Let $G$ be the transvection group of $M$, i.e., the identity component of the group generated by all $\sigma_{x}, x \in M$. Then $G$ is a Lie group which acts transitively on $M$. Hence we can write $M=G / H$ for some closed subgroup $H \subset G$, and we call this symmetric space irreducible if the isotropy representation of $H$ on $T_{x_{0}} M$ is irreducible, where $x_{0}:=e H \in M$. It follows that there is an involution

$$
\sigma_{0}:=A d_{\sigma_{x_{0}}}: G \longrightarrow G,
$$

and $H \subset G$ is $\sigma_{0}$-invariant, and its Lie algebra $\mathfrak{h} \subset \mathfrak{g}$ is the $(+1)$ - eigenspace of the differential $\mathrm{d} \sigma_{0}: \mathfrak{g} \rightarrow \mathfrak{g}$. Thus, Proposition 2.9 applies to $G / H$, and in fact the connection defined there coincides with the given connection on $M$.

Theorem 3.1 [32] Let $(M, \nabla)$ be an irreducible symmetric space with transvection group $G$, and let $\mathfrak{g}$ denote its Lie algebra. Then

1. G is semi-simple.

2. If $\mathfrak{g}=\mathfrak{h} \oplus \mathfrak{m}$ is the symmetric decomposition, then $[\mathfrak{m}, \mathfrak{m}]=\mathfrak{h}$.

3. The holonomy of the connection satisfies $H^{0} \subset H o l \subset H$, i.e., up to connected components, the isotropy group and the holonomy group coincide.

We should remark here that Cartan proved Theorem 3.1 only in the case of irreducible Riemannian symmetric spaces. However, his proof can be adapted to the general case immediately; see e.g. [47]. 
In fact, Cartan even succeeded in providing a classification of simply connected irreducible Riemannian symmetric spaces ([32]), i.e., those symmetric spaces whose holonomy group is contained in the orthogonal group. In this case, irreducibility and indecomposability are equivalent.

Later, M.Berger ([10]) gave a classification of simply connected affine symmetric spaces with irreducible holonomy. These are all pseudo-Riemannian since the Killing form of $\mathfrak{g}$ induces a non-degenerate inner product on $\mathfrak{m}$ which induces a pseudo-Riemannian metric.

In general, the classification of affine symmetric spaces is far from complete. In the case of pseudo-Riemannian symmetric spaces, this classification was established for metrics of signature $(1, n)$ by M.Cahen and N.Wallach ([27]), and recently by I.Kath and M.Olbrich for signatures $(2, n)$ ([52]). They also give a general construction method for such spaces of arbitrary signature.

\subsection{Holonomy of Riemannian manifolds}

Let $(M, g)$ be a Riemannian manifold. In this case, the holonomy group is contained in the orthogonal group $O(n)$ or, equivalently, its identity component is compact. Moreover, indecomposability is equivalent to irreducibility of the group. As we mentioned in section 3.1, the Riemannian symmetric spaces are classified by Cartan ([32]). Thus, Theorems 2.8 and 2.12 imply that it suffices to classify all irreducible non-symmetric Berger algebras which are contained in $O(n)$. This has been achieved by Berger ([9]) where the below classification table was established.

Another important question is the determination of parallel spinors, i.e., parallel sections of the spinor bundle of a spin manifold $M$. If we assume that the holonomy of $M$ is connected (e.g. if $M$ is simply connected, cf. Proposition 2.3), then the space of parallel spinors corresponds to the subspace of the spinor representation on which the holonomy algebra $\mathfrak{h o l}(M) \subset \mathfrak{s o}(n) \cong \mathfrak{s p i n}(n)$ acts trivially. These spaces have been described by M.Wang ([68]) for all entries in Berger's list, and we add the dimension of the space of parallel spinors for each of the holonomies in question.

Table 1:

CLASSIFICATION OF CONNECTED IRREDUCIBLE NON-SYMMETRIC HOLONOMIES CONTAINED IN $\mathrm{SO}(n)$

\begin{tabular}{|c|c|c|c|}
\hline$n$ & $\mathrm{H}$ & associated geometry & dim. of space of parallel spinors \\
\hline \hline$n \geq 2$ & $\mathrm{SO}(n)$ & generic Riemannian manifold & 0 \\
\hline $2 m \geq 4$ & $\mathrm{U}(m)$ & generic Kähler manifold & 0 \\
$2 m \geq 4$ & $\mathrm{SU}(m)$ & special Kähler manifold & 2 \\
\hline $4 m \geq 8$ & $\mathrm{Sp}(m) \cdot \mathrm{Sp}(1)$ & quaternionic Kähler manifold & 0 \\
$4 m \geq 8$ & $\mathrm{Sp}(m)$ & hyper-Kähler manifold & $m+1$ \\
\hline 7 & $\mathrm{G}_{2}$ & exceptional holonomy & 1 \\
8 & $\mathrm{Spin}(7)$ & exceptional holonomy & 1 \\
\hline
\end{tabular}

It was noted immediately that this list is contained in the list of subgroups of the orthogonal group which act transitively on the unit sphere. This fact was later proven directly by J.Simons ([66]) in an algebraic way. Recently, C.Olmos gave a beautiful simple argument showing this transitivity using only submanifold theory ([59]).

As it turns out, all of the groups in Table 1 do occur as holonomy of Riemannian connections.

1. $S O(n)$ is the reduced holonomy of a "generic" Riemannian manifold. 
2. If $H o l \subset U(m)$, then the metric $g$ is called Kähler. Kähler metrics form a natural class of complex manifolds, and the "generic" Kähler manifold has holonomy equal to $U(m)$.

3. If $\mathrm{Hol} \subset S U(m)$ then the metric is called a Calabi-Yau metric. Since $S U(m) \subset U(m)$, each Calabi-Yau metric is necessarily Kähler. In fact, a Kähler metric with connected holonomy group is Calabi-Yau if and only if its Ricci curvature vanishes.

The first examples of complete Calabi-Yau metrics were given by E.Calabi ([28]). Later, S.T.Yau's solution to the Calabi conjecture ([72]) showed that a compact Kähler manifold with trivial canonical line bundle or, equivalently, with vanishing first Chern class admits a unique Calabi-Yau metric whose Kähler form represents the same cohomology class as the Kähler form of the original Kähler metric. For explicit examples, we refer to the books by A.Besse ([11]), S.Salamon ([61]) and D.Joyce ([50]).

4. Metrics with $\mathrm{Hol}=S p(m) \cdot S p(1)$ are called quaternionic Kähler, although this terminology is somewhat misleading: quaternionic Kähler manifolds are not Kähler, as $S p(1) \cdot S p(m)$ is not contained in $U(m)$. Quaternionic Kähler manifolds are always Einstein, but not Ricci flat.

Homogeneous quaternionic-Kähler manifolds were classified by D.Aleksevskii and V.Cortés ([1], [36]). For more details on the theory of these manifolds, see [42, 43, 61, 62]. It is worth pointing out that there are so far no known examples of closed quaternionic Kähler manifolds with positive scalar curvature other than quaternionic projective space.

5. Metrics with $\mathrm{Hol} \subset S p(m)$ are called hyper-Kähler. These metrics are Kähler as $S p(m) \subset$ $S U(2 m)$. In fact, hyper-Kähler metrics admit a whole two-sphere worth of Kähler structures which induce the quaternionic structure. First explicit examples were found by Calabi ([28]). Compact examples were constructed using Yau's proof of the Calabi conjecture, see [6] for details.

6. The holonomy groups $G_{2}$ and $\operatorname{Spin}(7)$ are called exceptional holonomies as they only occur in dimension 7 and 8, respectively. The existence of metrics with exceptional holonomy was shown locally by R.Bryant ([16]). Complete examples were given by Bryant and Salamon ([22]), and compact examples were given by Joyce ([48, 49]) . See also [50] for a more detailed exposition.

Note that in general, the holonomy group of a Riemannian manifold may be non-compact as it may have infinitely many connected components. For example, let $M:=\mathbb{R}^{3} / \Gamma$, where $\mathbb{R}^{3}$ is equipped with the standard flat connection and $\Gamma$ is the cyclic group generated by an affine map whose linear part is rotation around an axis with irrational rotation angle and whose translation part is in direction of this axis. Then the holonomy of $M$ is a cyclic group whose closure is $S O(2) \subset O(3)$. Thus, the holonomy group is non-compact. However, note that $M$ is non-compact either.

It remained an open question for a long time whether the holonomy of a compact Riemannian manifold is necessarily compact (where the connection considered here is of course the Levi-Civita connection). In fact, it was conjectured in [11] that this is the case. 
However, as it turns out, the answer is negative. In fact, B.Wilking ([69]) constructed examples of compact Riemannian manifolds with noncompact holonomy. He also showed that any such manifold must be finitely covered by a torus bundle over a compact manifold, where the dimension of the torus fiber is at least four.

\subsection{Holonomy groups of Pseudo-Riemannian manifolds}

\subsubsection{Irreducible holonomy groups}

In [9], Berger also classified all connected irreducible Berger groups which are subgroups of $S O(p, q)$ which are therefore candidates for the holonomy group of a pseudo-Riemanian manifold with a metric of signature $(p, q)$. There were some minor omissions and errata on his list which were corrected by Bryant ([18]). As in the case of Riemannian holonomies, one can obtain the dimension of the space of parallel spinors in each of these cases which has been worked out by H.Baum and I.Kath ([5]). Summarizing all these results, we obtain the following table:

Table 2: CLASSIFICATION OF CONNECTED IRREDUCIBLE NON-SYMMETRIC HOLONOMIES CONTAINED IN $\mathrm{SO}_{0}(r, s)$

\begin{tabular}{|c|c|c|c|}
\hline$n=r+s$ & $\mathrm{H}$ & associated geometry & Dim. of space of parallel spinors \\
\hline \hline$p+q \geq 2$ & $\mathrm{SO}_{0}(p, q)$ & generic & 0 \\
$2 p \geq 4$ & $\mathrm{SO}(p, \mathbb{C})$ & generic complex & 0 \\
\hline $2(p+q) \geq 4$ & $\mathrm{U}(p, q)$ & pseudo-Kähler & 2 \\
$2(p+q) \geq 4$ & $\mathrm{SU}(p, q)$ & special pseudo-Kähler & $p+q+1$ \\
\hline $4(p+q) \geq 8$ & $\mathrm{Sp}(p, q)$ & pseudo-hyper-Kähler & 0 \\
\hline $4(p+q) \geq 8$ & $\mathrm{Sp}(p, q) \cdot \mathrm{Sp}(1)$ & pseudo-quaternionic Kähler & 0 \\
$4 p \geq 8$ & $\mathrm{Sp}(p, \mathbb{R}) \cdot \mathrm{SL}(2, \mathbb{R})$ & & 0 \\
$8 p \geq 16$ & $\mathrm{Sp}(p, \mathbb{C}) \cdot \mathrm{SL}(2, \mathbb{C})$ & & 1 \\
\hline 7 & $\mathrm{G}_{2}$ & & 2 \\
\hline 7 & $\mathrm{G}_{2}^{\prime}$ & & 1 \\
\hline 14 & $\mathrm{G}_{2}^{\mathbb{C}}$ & & 1 \\
\hline 8 & $\mathrm{Spin}(7)$ & & 1 \\
\hline
\end{tabular}

We should also point out that all of these Berger groups do occur as the holonomy of pseudoRiemannian manifolds, as has been shown by Bryant ([18]).

\subsubsection{Indecomposable Lorentzian holonomy groups}

A Lorentzian manifold is a pseudo-Riemannian manifold of signature $(n, 1)$. According to Berger's classification in Table 2, there is no proper irreducible subgroup of $S O_{0}(n, 1)$ which can occur as the holonomy group of a Lorentzian manifold. In fact, there is no proper irreducible subgroup of $S O_{0}(n, 1)$ at all - this fact has been shown in a purely geometric manner by Di Scala and Olmos ([38]). 
Therefore, we may assume that the holonomy representation is indecomposable but not irreducible. This implies that there must be a one-dimensional Hol-invariant subspace $\mathbb{R} \xi \subset \mathbb{R}^{n, 1}$ with $\xi \neq 0$ such that $\langle\xi, \xi\rangle=0$. Let $\Xi:=(\mathbb{R} \xi)^{\perp} /(\mathbb{R} \xi)$ which is well defined as $\mathbb{R} \xi \subset(\mathbb{R} \xi)^{\perp}$. Since $\mathrm{Hol}$ leaves $\mathbb{R} \xi$ and hence its orthogonal complement invariant, it follows that there is an induced action of $\mathrm{Hol}$ on $\Xi$ which preserves the induced positive definite inner product. (Note that this action may fail to be irreducible).

Based on work of L.Bérard-Bergery and A.Ikemakhen ([7]), the following classification result was established by T.Leistner.

Theorem 3.2 [55] Let $H \subset S O_{0}(n, 1)$ be a connected indecomposable, but not irreducible subgroup, and let $\hat{H} \subset S O(\Xi)$ be the image of the induced representation described above. Then the following are equivalent.

1. H is a Berger group,

2. $\hat{H}$ is a Berger group, i.e., it is either the isotropy group of an irreducible Riemannian symmetric space, or is one of the entries of Table 1, or the direct product of such groups.

Moreover, if a Lorentzian Spin-manifold admits a parallel spinor, then $H=\hat{H} \ltimes \mathbb{R}^{n}$, and the dimension of the space of parallel spinors coincides with this dimension for a Riemannian Spinmanifold with holonomy $\hat{H}$.

Furthermore, each indecomposable Berger group which is contained in $\mathrm{SO}_{0}(n, 1)$ does occur as the holonomy group of a Lorentzian manifold ([40, 55]).

\subsubsection{Indecomposable holonomy groups of Pseudo-Riemannian manifolds of signature $(p, q)$ with $p, q \geq 2$}

In the non-Lorentzian case, there are a number of results which we shall not describe here in more detail. We already mentioned the partial classification of symmetric spaces ([52]); another striking result is the classification of Kählerian holonomies of complex signature $(1, n)$ (hence of real signature $(2,2 n)$ ) by Galaev ([41]). Further results on signature $(2, n)$ may be found e.g. in $[39,45]$, and for split signature $(n, n)$ e.g. in [8].

\subsection{Special Symplectic Holonomy groups}

A symplectic connection is a torsion free connection on a symplectic manifold $(M, \omega)$ such that $\omega$ is parallel or, equivalently, $\mathrm{Hol} \subset S p(n, \mathbb{R})$. We say that this connection has special symplectic holonomy if $\mathrm{Hol}$ acts absolutely irreducibly on the tangent space or, equivalently, if $\mathrm{Hol}$ acts irreducibly and does not preserve any complex structure.

First special symplectic holonomies were given by Bryant ([17]) and by Q.-S.Chi, S.Merkulov and the author $([34,35])$. Finally, these holonomies were classified by Merkulov and the author ([56], see also [65]), and the possible holonomies are listed in Table 3. 
Table 3: Special Symplectic Holonomy Groups

\begin{tabular}{|c|c||c|c|}
\hline Group H & Representation space & Group H & Representation space \\
\hline \hline $\mathrm{SL}(2, \mathbb{R})$ & $\mathbb{R}^{4} \simeq \odot^{3} \mathbb{R}^{2}$ & $\mathrm{E}_{7}^{5}$ & $\mathbb{R}^{56}$ \\
$\mathrm{SL}(2, \mathbb{C})$ & $\mathbb{C}^{4} \simeq \odot^{3} \mathbb{C}^{2}$ & $\mathrm{E}_{7}^{7}$ & $\mathbb{R}^{56}$ \\
$\mathrm{SL}(2, \mathbb{R}) \cdot \mathrm{SO}(p, q)$ & $\mathbb{R}^{2(p+q)} \simeq \mathbb{R}^{2} \otimes \mathbb{R}^{p+q}, p+q \geq 3$ & $\mathrm{E}_{7}^{\mathbb{C}}$ & $\mathbb{C}^{56}$ \\
$\mathrm{SL}(2, \mathbb{C}) \cdot \mathrm{SO}(n, \mathbb{C})$ & $\mathbb{C}^{2 n} \simeq \mathbb{C}^{2} \otimes \mathbb{C}^{n}, n \geq 3$ & $\mathrm{Spin}(2,10)$ & $\mathbb{R}^{32}$ \\
$\mathrm{Sp}(1) \cdot \mathrm{SO}(n, \mathbb{H})$ & $\mathbb{H}^{n} \simeq \mathbb{R}^{4 n}, n \geq 2$ & $\mathrm{Spin}(6,6)$ & $\mathbb{R}^{32}$ \\
$\mathrm{SL}(6, \mathbb{R})$ & $\mathbb{R}^{20} \simeq \Lambda^{3} \mathbb{R}^{6}$ & $\mathrm{Spin}(6, \mathbb{H})$ & $\mathbb{R}^{32}$ \\
$\mathrm{SU}(1,5)$ & $\mathbb{R}^{20} \subset \Lambda^{3} \mathbb{C}^{6}$ & $\mathrm{Spin}(12, \mathbb{C})$ & $\mathbb{C}^{32}$ \\
$\mathrm{SU}(3,3)$ & $\mathbb{R}^{20} \subset \Lambda^{3} \mathbb{C}^{6}$ & $\mathrm{Sp}(3, \mathbb{R})$ & $\mathbb{R}^{14} \subset \Lambda^{3} \mathbb{R}^{6}$ \\
$\mathrm{SL}(6, \mathbb{C})$ & $\mathbb{C}^{20} \simeq \Lambda^{3} \mathbb{C}^{6}$ & $\mathrm{Sp}(3, \mathbb{C})$ & $\mathbb{C}^{14} \subset \Lambda^{3} \mathbb{C}^{6}$ \\
\hline
\end{tabular}

As it turns out, all of these holonomies share striking rigidity properties which we shall explain in more detail in section 4 .

\subsection{Irreducible Holonomy groups}

Holonomy groups which are irreducible, but of none of the above types, i.e., which preserve neither a (pseudo-)Riemannian nor a symplectic structure, have been investigated already by Berger ([9]) and later by Bryant $([18,19])$. A complete classification was obtained by Merkulov and the author ([56], see also [65]). We shall not deal much with the geometric content of these holonomies here, but rather conclude this survey with the classification table, referring the interested reader to the cited references. 
Table 4: LIST OF NON-RIEMANNIAN, NON-SYMPLECTIC HOLONOMY GROUPS

NOTATIONS: $\quad T_{\mathbb{F}}$ denotes any connected subgroup of $\mathbb{F}^{*}$.

$\odot^{p} V$ denotes the symmetric tensors of $V$ of degree $p$.

\begin{tabular}{|c|c|c|}
\hline Group H & Representation space $V$ & $\begin{array}{l}\text { restrictions } \\
\text { remarks }\end{array}$ \\
\hline $\mathrm{T}_{\mathbb{R}} \cdot \mathrm{SL}(n, \mathbb{C})$ & $\left\{A \in M_{n}(\mathbb{C}) \mid A=A^{*}\right\}$ & $n \geq 3$ \\
\hline$T_{\mathbb{R}} \cdot \operatorname{SL}(n, \mathbb{R}) \cdot \operatorname{SL}(m, \mathbb{R})$ & $\mathbb{R}^{n} \otimes \mathbb{R}^{m}$ & $n \geq m \geq 2, n m \neq 4$ \\
\hline$T_{\mathbb{R}} \cdot \operatorname{SL}(n, \mathbb{H}) \cdot \operatorname{SL}(m, \mathbb{H})$ & $\mathbb{H}^{n} \otimes_{\mathbb{H}} \mathbb{H}^{m}$ & $n \geq m \geq 1, n m \neq 1$ \\
\hline$T_{\mathbb{C}} \cdot \operatorname{SL}(n, \mathbb{C}) \cdot \operatorname{SL}(m, \mathbb{C})$ & $\mathbb{C}^{n} \otimes \mathbb{C}^{m}$ & $n \geq m \geq 2, n m \neq 4$ \\
\hline$T_{\mathbb{R}} \cdot \operatorname{SL}(n, \mathbb{R})$ & $\mathbb{R}^{n}$ & $n \geq 2$ \\
\hline$T_{\mathbb{R}} \cdot \operatorname{SL}(n, \mathbb{H})$ & $\mathbb{H}^{n}$ & $n \geq 1$ \\
\hline$T_{\mathbb{C}} \cdot \operatorname{SL}(n, \mathbb{R})$ & $\mathbb{C}^{n}$ & $n \geq 2$ \\
\hline$T_{\mathbb{C}} \cdot \operatorname{SL}(n, \mathbb{C})$ & $\mathbb{C}^{n}$ & $n \geq 2$ \\
\hline $\mathrm{U}(p, q)$ & $\mathbb{C}^{p+q}$ & $p+q \geq 2$ \\
\hline $\mathrm{SU}(p, q)$ & $\mathbb{C}^{p+q}$ & $p+q \geq 2, p q \neq 1$ \\
\hline$T_{\mathbb{C}} \cdot \mathrm{SU}(p, q)$ & $\mathbb{C}^{2}$ & $p+q=2$ \\
\hline$T_{\mathbb{R}} \cdot \operatorname{SL}(n, \mathbb{R})$ & $\Lambda^{2} \mathbb{R}^{n}$ & $n \geq 5$ \\
\hline$T_{\mathbb{C}} \cdot \operatorname{SL}(n, \mathbb{C})$ & $\Lambda^{2} \mathbb{C}^{n}$ & $n \geq 5$ \\
\hline$T_{\mathbb{R}} \cdot \operatorname{SL}(n, \mathbb{H})$ & $\left\{A \in M_{n}(\mathbb{H}) \mid A=A^{*}\right\}$ & $n \geq 3$ \\
\hline$T_{\mathbb{R}} \cdot \operatorname{SL}(n, \mathbb{R})$ & $\odot^{2} \mathbb{R}^{n}$ & $n \geq 3$ \\
\hline$T_{\mathbb{C}} \cdot \operatorname{SL}(n, \mathbb{C})$ & $\odot^{2} \mathbb{C}^{n}$ & $n \geq 3$ \\
\hline$T_{\mathbb{R}} \cdot \operatorname{SL}(n, \mathbb{H})$ & $\left\{A \in M_{n}(\mathbb{H}) \mid A=-A^{*}\right\}$ & $n \geq 2$ \\
\hline$T_{\mathbb{R}} \cdot \mathrm{SO}(p, q)$ & $\mathbb{R}^{p+q}$ & $p+q \geq 3$ \\
\hline$T_{\mathbb{C}} \cdot \mathrm{SO}(n, \mathbb{C})$ & $\mathbb{C}^{n}$ & $n \geq 3$ \\
\hline$T_{\mathbb{R}} \cdot \operatorname{Spin}(5,5)$ & $\Delta_{(5,5)}^{+}$ & \\
\hline$T_{\mathbb{R}} \cdot \operatorname{Spin}(1,9)$ & $\Delta_{(1,9)}^{+}$ & \\
\hline$T_{\mathbb{C}} \cdot \operatorname{Spin}(10, \mathbb{C})$ & $\left(\Delta_{10}^{+}\right)^{\mathbb{C}}$ & \\
\hline$T_{\mathbb{R}} \cdot \mathrm{E}_{6}^{1}$ & $\mathbb{R}^{27}$ & \\
\hline$T_{\mathbb{R}} \cdot \mathrm{E}_{6}^{4}$ & $\mathbb{R}^{27}$ & \\
\hline$T_{\mathbb{C}} \cdot \mathrm{E}_{6}^{\mathbb{C}}$ & $\mathbb{C}^{27}$ & \\
\hline $\mathrm{SL}(2, \mathbb{R}) \cdot \mathrm{SO}(p, q)$ & $\mathbb{R}^{2} \otimes \mathbb{R}^{p+q}$ & $p+q \geq 3$ \\
\hline $\operatorname{Sp}(1) \cdot \operatorname{SO}(n, \mathbb{H})$ & $\mathbb{H}^{n}$ & $n \geq 2$ \\
\hline $\operatorname{Sp}(n, \mathbb{R})$ & $\mathbb{R}^{2 n}$ & $n \geq 2$ \\
\hline $\mathbb{R}^{*} \cdot \operatorname{Sp}(2, \mathbb{R})$ & $\mathbb{R}^{4}$ & \\
\hline $\operatorname{Sp}(p, q)$ & $\mathbb{H}^{p+q}$ & $p+q \geq 2$ \\
\hline $\begin{array}{l}T_{\mathbb{R}} \cdot \operatorname{SL}(2, \mathbb{R}) \\
T_{\mathbb{C}} \cdot \operatorname{SL}(2, \mathbb{C})\end{array}$ & $\begin{array}{l}\odot^{3} \mathbb{R}^{2} \\
\odot^{3} \mathbb{C}^{2}\end{array}$ & \\
\hline
\end{tabular}




\section{Special symplectic connections}

In this section, we shall deal with the notion of a special symplectic connection in the sense of [26]. First of all, if $(M, \omega)$ is a symplectic manifold, then a symplectic connection is defined to be a torsion free connection such that $\omega$ is parallel or, equivalently, such that the holonomy of the connection is contained in the symplectic group

$$
\operatorname{Sp}(V, \omega):=\{g \in \operatorname{Aut}(V) \mid \omega(g x, g y)=\omega(x, y) \text { for all } x, y \in V\} \text {, }
$$

whose Lie algebra is given as

$$
\mathfrak{s p}(V, \omega):=\{h \in \operatorname{End}(V) \mid \omega(h x, y)+\omega(x, h y)=0 \text { for all } x, y \in V\} .
$$

A special symplectic connection is defined as a symplectic connection on a manifold of dimension at least 4 which belongs to one of the following classes.

\section{Bochner-Kähler and Bochner-bi-Lagrangian connections}

If the symplectic form is the Kähler form of a (pseudo-)Kähler metric, then its curvature decomposes into the Ricci curvature and the Bochner curvature ([13]). If the latter vanishes, then (the Levi-Civita connection of) this metric is called Bochner-Kähler.

Similarly, if the manifold is equipped with a bi-Lagrangian structure, i.e., two complementary Lagrangian distributions, then the curvature of a symplectic connection for which both distributions are parallel decomposes into the Ricci curvature and the Bochner curvature. Such a connection is called Bochner-bi-Lagrangian if its Bochner curvature vanishes.

For results on Bochner-Kähler and Bochner-bi-Lagrangian connections, see [20] and [51] and the references cited therein.

\section{Connections of Ricci type}

Under the action of the symplectic group, the curvature of a symplectic connection decomposes into two irreducible summands, namely the Ricci curvature and a Ricci flat component. If the latter component vanishes, then the connection is said to be of Ricci type.

Connections of Ricci type are critical points of a certain functional on the moduli space of symplectic connections ([15]). Furthermore, the canonical almost complex structure on the twistor space induced by a symplectic connection is integrable if and only if the connection is of Ricci type ([57, 67]). For further properties see also [3, 23, 24, 25].

\section{Connections with special symplectic holonomy}

A symplectic connection is said to have special symplectic holonomy if its holonomy is contained in a proper absolutely irreducible subgroup of the symplectic group. Thus, these are connections whose holonomy is listed in Table 3 on page 23.

The special symplectic holonomies have been classified in [56] and further investigated in $[17,34,63,64,65]$. 
We can consider all of these conditions also in the complex case, i.e., for complex manifolds of complex dimension at least 4 with a holomorphic symplectic form and a holomorphic connection.

At first, it may seem unmotivated to collect all these structures in one definition, but we shall provide ample justification for doing so. Indeed, there is a beautiful link between special symplectic connections and parabolic contact geometry.

For this, consider a simple Lie group $G$ with Lie algebra $\mathfrak{g}$. We say that $\mathfrak{g}$ is 2 -gradable, if $\mathfrak{g}$ contains the root space of a long root. This is equivalent to saying that there is a decomposition as a graded vector space

$$
\mathfrak{g}=\mathfrak{g}^{-2} \oplus \mathfrak{g}^{-1} \oplus \mathfrak{g}^{0} \oplus \mathfrak{g}^{1} \oplus \mathfrak{g}^{2}, \quad \text { and } \quad\left[\mathfrak{g}^{i}, \mathfrak{g}^{j}\right] \subset \mathfrak{g}^{i+j},
$$

with $\operatorname{dim} \mathfrak{g}^{ \pm 2}=1$. Indeed, there is a (unique) element $H_{\alpha_{0}} \in\left[\mathfrak{g}^{-2}, \mathfrak{g}^{2}\right] \subset \mathfrak{g}^{0}$ such that $\mathfrak{g}^{i}$ is the eigenspace of $a d\left(H_{\alpha_{0}}\right)$ with eigenvalue $i=-2, \ldots, 2$, and any non-zero element of $\mathfrak{g}^{ \pm 2}$ is a long root vector.

Denote by $\mathfrak{p}:=\mathfrak{g}^{0} \oplus \mathfrak{g}^{1} \oplus \mathfrak{g}^{2} \leq \mathfrak{g}$ and let $P \subset \mathrm{G}$ be the corresponding connected Lie subgroup. It follows that the homogeneous space $\mathscr{C}:=\mathrm{G} / P$ carries a canonical G-invariant contact structure which is determined by the $A d_{P}$-invariant distribution $\mathfrak{g}^{-1} \bmod \mathfrak{p} \subset \mathfrak{g} / \mathfrak{p} \cong T \mathscr{C}$. In fact, we may regard $\mathscr{C}$ as the projectivisation of the adjoint orbit of a maximal root vector. That is, we view $\mathscr{C} \subset \mathbb{P}^{o}(\mathfrak{g})$ where $\mathbb{P}^{O}(V)$ denotes the set of oriented lines through 0 of a vector space $V$, so that $\mathbb{P}^{o}(V)$ is the sphere if $V$ is real, and $\mathbb{P}^{o}(V)$ is a complex projective space if $V$ is complex.

Each $a \in \mathfrak{g}$ induces an action field $a^{*}$ on $\mathscr{C}$ with flow $\mathrm{T}_{a}:=\exp (\mathbb{R} a) \subset \mathrm{G}$ which hence preserves the contact structure on $\mathscr{C}$. Let $\mathscr{C}_{a} \subset \mathscr{C}$ be the open subset on which $a^{*}$ is transversal to the contact distribution. There is a unique contact form $\alpha \in \Omega^{1}\left(\mathscr{C}_{a}\right)$ such that $\alpha\left(a^{*}\right) \equiv 1$. That is, $a^{*}$ is a Reeb vector field of the contact form $\alpha$.

We can cover $\mathscr{C}_{a}$ by open sets $U$ such that the local quotient $M_{U}:=\mathrm{T}_{a}^{l o c} \backslash U$, i.e., the quotient of $U$ by a sufficiently small neighbourhood of the identity in $\mathrm{T}_{a}$, is a manifold. Then $M_{U}$ inherits a canonical symplectic structure $\omega \in \Omega^{2}\left(M_{U}\right)$ such that $\pi^{*}(\omega)=\mathrm{d} \alpha$ for the canonical projection $\pi: U \rightarrow M_{U}$.

It is now our aim to construct a connection on $M_{U}$ which is 'naturally' associated to the given structure. For this, we let $G_{0} \subset G$ be the connected subgroup with Lie algebra $\mathfrak{g}^{0} \leq \mathfrak{g}$. Since $\mathfrak{g}^{0} \leq \mathfrak{p}$ and hence $\mathrm{G}_{0} \subset P$, it follows that we have a fibration

$$
P / \mathrm{G}_{0} \longrightarrow \mathrm{G} / \mathrm{G}_{0} \longrightarrow \mathscr{C}=\mathrm{G} / P \text {. }
$$

In fact, we may interpret $\mathrm{G} / \mathrm{G}_{0}:=\left\{(\alpha, v) \in T_{p}^{*} \mathscr{C} \times T_{p} \mathscr{C} \mid p \in \mathscr{C}, \alpha\left(\mathscr{D}_{p}\right)=0, \alpha(v)=1\right\}$, where $\mathscr{D} \subset T \mathscr{C}$ denotes the contact distribution. Thus, given $a \in \mathfrak{g}$, then for each $p \in \mathscr{C}_{a}$ we may regard the pair $\left(\alpha_{p}, a_{p}^{*}\right)$ from above as a point in $\mathrm{G} / \mathrm{G}_{0}$, i.e., we have a canonical embedding $\imath: \mathscr{C}_{a} \hookrightarrow$ $\mathrm{G} / \mathrm{G}_{0}$.

Let $\Gamma_{a}:=\pi^{-1}\left(\imath\left(\mathscr{C}_{a}\right)\right) \subset \mathrm{G}$ where $\pi: \mathrm{G} \rightarrow \mathrm{G} / \mathrm{G}_{0}$ is the canonical projection. Then the restriction $\pi: \Gamma_{a} \rightarrow \imath\left(\mathscr{C}_{a}\right) \cong \mathscr{C}_{a}$ becomes a principal $\mathrm{G}_{0}$-bundle.

Consider the Maurer-Cartan form $\mu:=g^{-1} \mathrm{~d} g \in \Omega^{1}(\mathrm{G}) \otimes \mathfrak{g}$ which we decompose according to (30) as $\mu=\sum_{i=-2}^{2} \mu_{i}$ with $\mu_{i} \in \Omega^{1}(\mathrm{G}) \otimes \mathfrak{g}^{i}$. Then we can show the following.

Proposition 4.1 [26] Let $a \in \mathfrak{g}$ be such that $\mathscr{C}_{a} \subset \mathscr{C}$ is non-empty, define the action field $a^{*} \in \mathfrak{X}(\mathscr{C})$ and the principal $G_{0}$-bundle $\pi: \Gamma_{a} \rightarrow \mathscr{C}_{a}$ with $\Gamma_{a} \subset G$ from above. Then we have the following. 
1. The restriction of the components $\mu_{0}+\mu_{-1}+\mu_{-2}$ of the Maurer-Cartan form to $\Gamma_{a}$ yields a pointwise linear isomorphism $T \Gamma_{a} \rightarrow \mathfrak{g}^{0} \oplus \mathfrak{g}^{-1} \oplus \mathfrak{g}^{-2}$.

2. There is a linear map $R: \mathfrak{g}^{0} \rightarrow \Lambda^{2}\left(\mathfrak{g}^{1}\right)^{*} \otimes \mathfrak{g}^{0}$ and a smooth function $\rho: \Gamma_{a} \rightarrow \mathfrak{g}^{0}$ with the following property. If we define the differential forms $\kappa \in \Omega^{1}\left(\Gamma_{a}\right), \theta \in \Omega^{1}\left(\Gamma_{a}\right) \otimes \mathfrak{g}^{1}$ and $\eta \in \Omega^{1}\left(\Gamma_{a}\right) \otimes \mathfrak{g}^{0}$ by the equation

$$
\mu_{0}+\mu_{-1}+\mu_{-2}=-2 \kappa\left(\frac{1}{2} e_{-2}+\rho\right)+\theta+\eta
$$

for a fixed element $0 \neq e_{-2} \in \mathfrak{g}^{-2}$, then the following equations hold:

$$
d \kappa=\frac{1}{2}\left\langle e_{-2},[\theta, \theta]\right\rangle
$$

where $\langle$,$\rangle refers to the Killing form of \mathfrak{g}$, and

$$
\begin{aligned}
& d \theta+\eta \wedge \theta=0 \\
& d \eta+\frac{1}{2}[\eta, \eta]=R_{\rho}(\theta \wedge \theta) .
\end{aligned}
$$

Since the Maurer-Cartan form and hence $\kappa, \theta$ and $\eta$ are invariant under the left action of the subgroup $\mathrm{T}_{a} \subset \mathrm{G}$, we immediately get the following

Corollary 4.2 [26] On $T_{a} \backslash \Gamma_{a}$, there is a coframing $\eta+\theta \in \Omega^{1}\left(T_{a} \backslash \Gamma_{a}\right) \otimes\left(\mathfrak{g}^{0} \oplus \mathfrak{g}^{1}\right)$ satisfying the structure equations (33) for a suitable function $\rho: T_{a} \backslash \Gamma_{a} \rightarrow \mathfrak{g}^{0}$.

Thus, we could, in principle, regard $\theta$ and $\eta$ as the tautological and the connection 1-form, respectively, of a connection on the principal bundle $\mathrm{T}_{a} \backslash \Gamma_{a} \rightarrow \mathrm{T}_{a} \backslash \Gamma_{a} / \mathrm{G}_{0}$ whose curvature is represented by $R_{\rho}$. However, $\mathrm{T}_{a} \backslash \Gamma_{a} / \mathrm{G}_{0} \cong \mathrm{T}_{a} \backslash \mathscr{C}_{a}$ will in general be neither Hausdorff nor locally Euclidean, so the notion of a principal bundle cannot be defined globally.

The way out of this difficulty is to consider local quotients only, i.e., we restrict to sufficiently small open subsets $U \subset \mathscr{C}_{a}$ for which the local quotient $T_{a}^{l o c} \backslash U$ is a manifold. Clearly, $\mathscr{C}_{a}$ can be covered by such open cells.

Moreover, if we describe explicitly the curvature endomorphisms $R_{\rho}$ for $\rho \in \mathfrak{g}^{0}$, then one can show that - depending on the choice of the 2-gradable simple Lie algebra $\mathfrak{g}$ - the connections constructed above satisfy one of the conditions for a special symplectic connection mentioned before.

More precisely, we have the following

Theorem 4.3 [26] Let $\mathfrak{g}$ be a simple 2-gradable Lie algebra with $\operatorname{dim} \mathfrak{g} \geq 14$, and let $\mathscr{C} \subset \mathbb{P}^{o}(\mathfrak{g})$ be the projectivisation of the adjoint orbit of a maximal root vector. Let $a \in \mathfrak{g}$ be such that $\mathscr{C}_{a} \subset \mathscr{C}$ is non-empty, and let $T_{a}=\exp (\mathbb{R} a) \subset G$. If for an open subset $U \subset \mathscr{C}_{a}$ the local quotient $M_{U}=T_{a}^{\text {loc }} \backslash U$ is a manifold, then $M_{U}$ carries a special symplectic connection. 
The dimension restriction on $\mathfrak{g}$ guarantees that $\operatorname{dim} M_{U} \geq 4$ and rules out the Lie algebras of type $A_{1}, A_{2}$ and $B_{2}$.

The type of special symplectic connection on $M_{U}$ is determined by the Lie algebra $\mathfrak{g}$. In fact, there is a one-to-one correspondence between the various conditions for special symplectic connections and simple 2-gradable Lie algebras. More specifically, if the Lie algebra $\mathfrak{g}$ is of type $A_{n}$, then the connections in Theorem 4.3 are Bochner-Kähler of signature $(p, q)$ if $\mathfrak{g}=\mathfrak{s u}(p+1, q+1)$ or Bochner-bi-Lagrangian if $\mathfrak{g}=\mathfrak{s l}(n, \mathbb{R})$; if $\mathfrak{g}$ is of type $C_{n}$, then $\mathfrak{g}=\mathfrak{s p}(n, \mathbb{R})$ and these connections are of Ricci-type; if $\mathfrak{g}$ is a 2-gradable Lie algebra of one of the remaining types, then the holonomy of $M_{U}$ is contained in one of the special symplectic holonomy groups in Table 3 on page 23. Also, for two elements $a, a^{\prime} \in \mathfrak{g}$ for which $\mathscr{C}_{a}, \mathscr{C}_{a^{\prime}} \subset \mathscr{C}$ are non-empty, the corresponding connections from Theorem 4.3 are equivalent if and only if $a^{\prime}$ is G-conjugate to a positive multiple of $a$.

If $\mathrm{T}_{a} \cong S^{1}$ then $\mathrm{T}_{a} \backslash \mathscr{C}_{a}$ is an orbifold which carries a special symplectic orbifold connection by Theorem 4.3. Hence it may be viewed as the "standard orbifold model" for (the adjoint orbit of) $a \in \mathfrak{g}$. For example, in the case of positive definite Bochner-Kähler metrics, we have $\mathscr{C} \cong S^{2 n+1}$, and for connections of Ricci-type, we have $\mathscr{C} \cong \mathbb{R} \mathbb{P}^{2 n+1}$. Thus, in both cases the orbifolds $\mathrm{T}_{a} \backslash \mathscr{C}$ are weighted projective spaces if $\mathrm{T}_{a} \cong S^{1}$, hence the standard orbifold models $\mathrm{T}_{a} \backslash \mathscr{C}_{a} \subset \mathrm{T}_{a} \backslash \mathscr{C}$ are open subsets of weighted projective spaces.

Surprisingly, the connections from Theorem 4.3 exhaust all special symplectic connections, at least locally. Namely we have the following

Theorem 4.4 [26] Let $(M, \omega)$ be a symplectic manifold with a special symplectic connection of class $C^{4}$, and let $\mathfrak{g}$ be the Lie algebra associated to the special symplectic condition as above.

1. Then there is a principal $\hat{T}$-bundle $\hat{M} \rightarrow M$, where $\hat{T}$ is a one dimensional Lie group which is not necessarily connected, and this bundle carries a principal connection with curvature $\omega$.

2. Let $T \subset \hat{T}$ be the identity component. Then there is an $a \in \mathfrak{g}$ such that $T \cong T_{a} \subset G$, and $a T_{a^{-}}$ equivariant local diffeomorphism $\hat{\imath}: \hat{M} \rightarrow \mathscr{C}_{a}$ which for each sufficiently small open subset $V \subset \hat{M}$ induces a connection preserving diffeomorphism $\iota: T^{l o c} \backslash V \rightarrow T_{a}^{l o c} \backslash U=M_{U}$, where $U:=\hat{\imath}(V) \subset \mathscr{C}_{a}$ and $M_{U}$ carries the connection from Theorem 4.3.

The situation in Theorem 4.4 can be illustrated by the following commutative diagram, where the vertical maps are quotients by the indicated Lie groups, and $\mathrm{T} \backslash \hat{M} \rightarrow M$ is a regular covering.

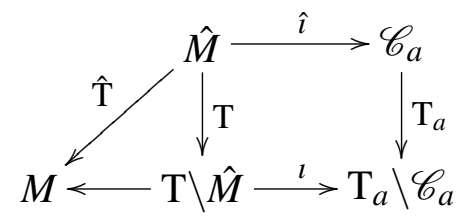

In fact, one might be tempted to summarize Theorems 4.3 and 4.4 by saying that for each $a \in \mathfrak{g}$, the quotient $\mathrm{T}_{a} \backslash \mathscr{C}_{a}$ carries a canonical special symplectic connection, and the map $\imath: \mathrm{T} \backslash \hat{M} \rightarrow$ $\mathrm{T}_{a} \backslash \mathscr{C}_{a}$ is a connection preserving local diffeomorphism. If $\mathrm{T}_{a} \backslash \mathscr{C}_{a}$ is a manifold or an orbifold, then this is indeed correct. In general, however, $\mathrm{T}_{a} \backslash \mathscr{C}_{a}$ may be neither Hausdorff nor locally Euclidean, hence one has to formulate these results more carefully. 
As consequences, we obtain the following

Corollary 4.5 All special symplectic connections of $C^{4}$-regularity are analytic, and the local moduli space of these connections is finite dimensional, in the sense that the germ of the connection at one point up to $3 \mathrm{rd}$ order determines the connection entirely. In fact, the generic special symplectic connection associated to the Lie algebra $\mathfrak{g}$ depends on $(r k(\mathfrak{g})-1)$ parameters.

Moreover, the Lie algebra $\mathfrak{s}$ of affine vector fields, i.e., vector fields on $M$ whose flow preserves the connection, is isomorphic to $\mathfrak{z}(a) /(\mathbb{R} a)$ with $a \in \mathfrak{g}$ from Theorem 4.4 , where $\mathfrak{z}(a)=\{x \in \mathfrak{g} \mid$ $[x, a]=0\}$. In particular, $\operatorname{dim} \mathfrak{s} \geq r k(\mathfrak{g})-1$ with equality implying that $\mathfrak{s}$ is abelian.

When counting the parameters in the above corollary, we regard homothetic special symplectic connections as equal, i.e., $(M, \omega, \nabla)$ is considered equivalent to $\left(M, e^{t_{0}} \omega, \nabla\right)$ for all $t_{0} \in \mathbb{R}$ or $\mathbb{C}$.

We can generalize Theorem 4.4 and Corollary 4.5 easily to orbifolds. Indeed, if $M$ is an orbifold with a special symplectic connection, then we can write $M=\hat{\mathrm{T}} \backslash \hat{M}$ where $\hat{M}$ is a manifold and $\hat{\mathrm{T}}$ is a one dimensional Lie group acting properly and locally freely on $\hat{M}$, and there is a local diffeomorphism $\hat{\imath}: \hat{M} \rightarrow \mathscr{C}_{a}$ with the properties stated in Theorem 4.4.

There is a remarkable similarity between the cones $\mathscr{C}_{i} \subset \mathfrak{g}_{i}, i=1,2$, for the simple Lie algebras $\mathfrak{g}_{1}:=\mathfrak{s u}(n+1,1)$ and $\mathfrak{g}_{2}:=\mathfrak{s p}(n, \mathbb{R})$. Namely, $\mathscr{C}_{1}=S^{2 n+1}$ with the standard $C R$-structure, and $\mathfrak{g}_{1}$ is the Lie algebra of the group $\mathrm{SU}(n+1,1)$ of $C R$-isomorphisms of $S^{2 n+1}$ ([51]). On the other hand, $\mathscr{C}_{2}=\mathbb{R P}^{2 n+1}$, regarded as the lines in $\mathbb{R}^{2 n+2}$ with the projectivised action of $\mathfrak{s p}(n+1, \mathbb{R})$ on $\mathbb{R}^{2 n+2}$. Thus, $\mathscr{C}_{1}$ is the universal cover of $\mathscr{C}_{2}$, so that the local quotients $\mathrm{T}_{a} \backslash \mathscr{C}_{a}$ are related. In fact, we have the following result.

Proposition 4.6 [60] Consider the action of the 2-gradable Lie algebras $\mathfrak{g}_{1}:=\mathfrak{s u}(n+1,1)$ and $\mathfrak{g}_{2}:=\mathfrak{s p}(n+1, \mathbb{R})$ on the projectivised orbits $\mathscr{C}_{1}$ and $\mathscr{C}_{2}$, respectively. Then the following are equivalent.

1. For $a_{i} \in \mathfrak{g}_{i}$ the actions of $T_{a_{i}} \subset G_{i}$ on $\mathscr{C}_{i}$ are conjugate for $i=1,2$,

2. $a_{i} \in \mathfrak{u}(n+1)$ where $\mathfrak{u}(n+1) \subset \mathfrak{g}_{i}$ for $i=1,2$ via the two standard embeddings.

This together with the preceding results yields the following

\section{Theorem 4.7 [60]}

1. Let $(M, \omega, \nabla)$ be a symplectic manifold with a connection of Ricci type, and suppose that the corresponding element $a \in \mathfrak{s p}(n+1, \mathbb{R})$ from Theorem 4.3 is conjugate to an element of $\mathfrak{u}(n+1) \subset \mathfrak{s p}(n+1, \mathbb{R})$. Then M carries a canonical Bochner-Kähler metric whose Kähler form is given by $\omega$.

2. Conversely, let $(M, \omega, J)$ be a Bochner-Kähler metric such that the element $a \in \mathfrak{s u}(n+1,1)$ from Theorem 4.4 is conjugate to an element of $\mathfrak{u}(n+1) \subset \mathfrak{s u}(n+1,1)$. Then $(M, \omega)$ carries a canonical connection of Ricci-type.

Note that in [20], Bochner-Kähler metrics have been locally classified. In this terminology, the Bochner-Kähler metrics in the above theorem are called Bochner-Kähler metrics of type I. For more details, we also refer the reader to [12]. 


\section{References}

[1] D. V. AlekseevskiI, Classification of quaternionic spaces with a transitive solvable group of motions, Math. USSR Izv. 9, 297-339 (1975)

[2] W. Ambrose, I. M. Singer, A Theorem on holonomy, Trans. Amer. Math. Soc. 75, 428-443 (1953)

[3] F.Baguis, M.CAhen, A construction of symplectic connections through reduction, Let.Math.Phys 57, 149-160 (2001)

[4] H. Baum, Eichfeldtheiorie, Springer Verlag (2009)

[5] H. BAum, I. Kath, Parallel spinors and holonomy groups on pseudo-Riemannian spin-manifolds, Ann. Glob. Anal. Geom. 17, (1), 1-17 (1999)

[6] A. Beauville, Variétés Kählériennes dont la première classe de Chern est nulle, Jour. Diff. Geom. 18, 755-782 (1983)

[7] L.Bérard-Bergery, A. Ikemakhen, On the holonomy of Lorentzian manifolds, Geometry in mathematical physics and related topics (Los Angeles, CA, 1990), Proc. Sympos. Pure Math., AMS, 54 (2), 27-40 (1993)

[8] L.BÉRARD-BERGERY, A. IKEMAKHEN, Sur l'holonomie des variétés pseudo- riemaniennes de signature (n,n), Bull.Soc.Math.France 125 (1), 93-114 (1997)

[9] M. BERGER, Sur les groupes d'holonomie des variétés à connexion affine et des variétés Riemanniennes, Bull. Soc. Math. France 83, 279-330 (1955)

[10] M. BERGER, Les espaces symétriques noncompacts, Ann.Sci.Ecol.Norm.Sup. 74, 85-177 (1957)

[11] A. L. Besse, Einstein Manifolds, Ergebnisse der Mathematik und ihrer Grenzgebiete, 3. Folge, Band 10, Springer-Verlag, Berlin, New York (1987)

[12] P. Bieliavsky, M. Cahen, S. Gutt, J. Rawnsley, L.J. Schwachhöfer, Symplectic Connections, International Journal of Geometric Methods in Modern Physics, 3 (3), 375 - 420 (2006)

[13] S. Bochner, Curvature and Betti numbers, II, Ann. Math. 5077 - 93 (1949)

[14] A. Borel, A. LiChnerowiCZ, Groupes d'holonomie des variétés riemanniennes, C.R.Acad.Sci.Paris 2341835 - 1837 (1952)

[15] F. Bourgeois, M. CAhen, A variational principle for symplectic connections, J.Geom.Phys 30, 233-265 (1999)

[16] R. BRyAnt, Metrics with exceptional holonomy, Ann. Math. 126, 525-576 (1987)

[17] R. Bryant, Two exotic holonomies in dimension four, path geometries, and twistor theory, Proc. Symp. in Pure Math. 53, 33-88 (1991)

[18] R. BRyAnt, Classical, exceptional, and exotic holonomies: a status report, Actes de la Table Ronde de Géométrie Différentielle en l'Honneur de Marcel Berger, Collection SMF Séminaires and Congrès 1 (Soc. Math. de France) 93-166, (1996). 
[19] R. BRYAnt, Recent Advances in the Theory of Holonomy, Séminaire Bourbaki, exposé no. 861, Paris: Société Mathématique de France, Ast érisque. 266, 351-374 (2000)

[20] R. BRYANT, Bochner-Kähler metrics, J. Amer. Math. Soc. 14623 - 715 (2001).

[21] R. BRYAnt, Geometry of manifolds with Special Holonomy: "100 years of Holonomy”, Cont.Math. 395, 29 -38 (2006)

[22] R. BRyAnt, S. SALAmon, On the construction of some complete metrics with exceptional holonomy, Duke. Math. Jour. 58, $829-850$ (1989)

[23] M. Cahen, S. Gutt, J. Rawnsley, Symmetric symplectic spaces with Ricci-type curvature, G.Dito, D.Sternheimer (ed.), Conférence Moshé Flato 1999, Vol.II, Math.Phys.Stud. 22, 81-91 (2000)

[24] M. Cahen, S. Gutt, J. Horowitz, J. Rawnsley, Homogeneous symplectic manifolds with Riccitype curvature, J.Geom.Phys. 38 140-151 (2001)

[25] M. Cahen, S. GutT, L.J. SchwachHöFer, Construction of Ricci-type connections by reduction and induction, in: "The Breadth of Symplectic and Poisson Geometry", Festschrift in Honour of Alan Weinstein, Birkhäuser Verlag, p. 41 - 58 (2004)

[26] M. Cahen, L.J. SchwachhöFer, Special Symplectic Connections, J.Diff.Geom. 83 (2), 229 - 271 (2009)

[27] M. Cahen, N. Wallach, Lorentzian symmetric spaces. Bull. Amer. Math. Soc. 76, 585-591 (1970)

[28] E. CAlabI, Métriques kähleriennes et fibrés holomorphes, Ann.Ec.Norm.Sup. 12, 269-294 (1979)

[29] É. CARTAn, Les groupes de transformations continus, infinis, simples, Ann. Éc. Norm. 26, 93-161 (1909)

[30] E. CARTAn, Sur les variétés à connexion affine et la théorie de la relativité généralisée I \& II, Ann.Sci.Ecol.Norm.Sup. 40, 325-412 (1923) et 41, 1-25 (1924) ou Oeuvres complètes, tome III, 659746 et $799-824$.

[31] E. Cartan, La géométrie des espaces de Riemann, Mémorial des Sciences Mathématiques, Paris, Gauthier-Villars, vol. 5 (1925)

[32] E. CARTAn, Sur une classe remarquable d'espaces de Riemann, Bull.Soc.Math.France 54, 214-264 (1926), 55, 114-134 (1927) ou Oeuvres complètes, tome I, vol. 2, 587-659.

[33] E. CARTAn, Les groupes d'holonomie des espaces généralisés, Acta.Math. 48, 1-42 (1926) ou Oeuvres complètes, tome III, vol. 2, 997-1038.

[34] Q.-S. Chi, S.A. Merkulov, L.J. Schwachhöfer, On the Existence of Infinite Series of Exotic Holonomies, Inv. Math. 126, 391-411 (1996)

[35] Q.-S. ChI, S.A. Merkulov, L.J. SCHWAChHÖFER, Exotic holonomies $E_{7}^{(a)}$, Int.Jour.Math. 8, 583594 (1997)

[36] V. CORTÉs, Alekseevskian spaces, Diff.Geom.Appl. 6, 129-168 (1996) 
[37] G. De Rham, Sur la réductibilité d'un espace de Riemann, Comm.Math.Helv. 26, 328-344 (1952)

[38] A. Di Scala, C. Olmos, The geometry of homogeneous submanifolds in hyperbolic space, Math. Zeit. 237 (1), 199-209 (2001)

[39] K. GALAEV, Remark on holonomy groups of pseudo-Riemannian manifolds of signature $(2, n+2)$, arXive: math.DG/0406397 (2004)

[40] A. Galaev, Metrics that realize all Lorentzian holonomy algebras, Int.J.Geom.Methods Mod. Phys., 3 (5-6), 1025-1045 (2006)

[41] K. GALAEV, Holonomy groups and special geometric structures of pseudo-K ählerian manifolds of index 2, arXive: math.DG/0612392 (2006)

[42] K. GALICKI, A generalization of the momentum mapping construction for quaternionic Kähler manifolds, Comm. Math. Phys. 108, 117-138 (1987)

[43] K. Galicki, H.B. LaWson, Quaternionic reduction and quaternionic orbifolds, Math. Ann. 282, $1-21(1988)$

[44] V. Guillemin, The integrability problem for G-structures, Trans. Amer. Math. Soc. 116, 544-560 (1965)

[45] A. IKeMAKHEN, Sur l'holonomie des variétés pseudo-riemaniennes de signature $(2,2+n)$, Publ.Math. 43 (1), 55-84 (1999)

[46] J. Hano, H. OzEKI, On the holonomy groups of linear connections, Nagoya Math. Jour. 10 97-100 (1956)

[47] S. Helgason, Differential Geometry, Lie Groups, and Symmetric Spaces, Graduate Studies in Mathematics, Vol. 34, AMS, Providence, RI, (2001).

[48] D. JoYCE, Compact 8-manifolds with holonomy $G_{2}:$ I \& II, Jour.Diff.Geo. 43, 291-375 (1996)

[49] D. JoyCE, Compact Riemannian 7-manifolds with holonomy Spin(7), Inv. Math. 123, 507-552 (1996)

[50] D. JoyCE, Compact manifolds with Special holonomy, Oxford Mathem. Monographs, Oxford Sci. Pub. (2000)

[51] Y. Kamishima, Uniformization of Kähler manifolds with vanishing Bochner tensor, Acta Math. 172 229 - 308 (1994)

[52] I. KATH, M. OlBRICH, On the structure of pseudo-Riemannian symmetric spaces. Transform.Groups 14 (4), 847-885 (2009)

[53] S. Kobayashi And K. NAgano, On filtered Lie algebras and geometric structures II J. Math. Mech. 14, 513-521 (1965)

[54] S. Kobayashi, K. Nomizu, Foundations of Differential Geometry, I, Interscience publishers (1963)

[55] T. LeISTnER, On the classification of Lorentzian holonomy groups Jour.Diff.Geo. 76 (3) 423 - 484 (2007) 
[56] S.A. Merkulov, L.J. SchwachHÖFER, Classification of irreducible holonomies of torsion free affine connections, Ann. Math. 150, 77-149 (1999); Addendum: Classification of irreducible holonomies of torsion-free affine connections, Ann. Math. 150, 1177-1179 (1999)

[57] N.R. O’Brian, J. RAWnSley, Twistor spaces, Ann.Glob.Anal.Geom 3, 29 - 58 (1985)

[58] T. OCHIAI, Geometry associated with semi-simple flat homogeneous spaces, Trans. Amer. Math. Soc. 152, 159-193 (1970)

[59] C. Olmos, A geometric proof of the Berger holonomy theorem, Ann. Math. 161 (1), 579-588 (2005)

[60] M. PANÁK, L. SCHWACHHÖFER, Bochner-Kähler metrics and connections of Ricci type, Proceedings of the 10th International Conference on Differential Geometry and Its Applications 2007, Differential geometry and its applications, 339-352 (2008)

[61] S. SAlamon, Riemannian geometry and holonomy groups, Pitman Research Notes in Mathematics, no. 201, Longman Scientific \& Technical, Essex (1989)

[62] S. Salamon, Quaternionic Kähler manifolds, Inv. Math. 67, 143-171 (1982)

[63] L.J. SchWACHHÖFER, On the classification of holonomy representations, Habilitationsschrift, Universität Leipzig (1998)

[64] L.J. SCHWACHHÖFER, Homogeneous connections with special symplectic holonomy, Math.Zeit. 238, $655-688(2001)$

[65] L.J. SCHWACHHÖFER, Connections with irreducible holonomy representations, Adv.Math. 160, 1 $80(2001)$

[66] J. Simons, On transitivity of holonomy systems, Ann. Math. 76, 213-234 (1962)

[67] I. VAISman, Variations on the theme of twistor spaces, Balkan J.Geom.Appl. 3135 - 156 (1998)

[68] M. WANG, Parallel spinors and parallel forms, Ann. Global Anal. Geom. 7 (1), 59-68 (1989)

[69] B. WILKING, On compact Riemannian manifolds with noncompact holonomy groups, J.Diff.Geom. 52 (2), 223-257 (1999)

[70] H. Wu, On the de Rham decomposition theorem, Illinois.J.Math. 8, 291-311 (1964)

[71] H. Wu, Holonomy groups of indefinite metrics, Pac.J.Math. 20 (2), 351-392 (1967)

[72] S.T. YAU, On the Ricci curvature of a compact Kähler mainfold and the complex Monge-Ampère equation I, Com.Pure and Appl. Math 31, 339-411 (1978) 


\section{Preprints ab 2008/15}

2010-06

2010-05

2010-04

2010-03

2010-02

2010-01

2009-16

2009-15

2009-14

2009-13

2009-12

2009-11

2009-10

2009-09

2009-08

2009-07

2009-06

2009-05
Lorenz J. Schwachhöfer

Holonomy Groups and Algebras

\section{Agnes Lamacz}

Dispersive effective models for waves in heterogeneous media

B. Schweizer and M. Veneroni

Periodic homogenization of Prandtl-Reuss plasticity equations in arbitrary dimension

Holger Dette and Karl Friedrich Siburg and Pavel A. Stoimenov

A copula-based nonparametric measure of regression dependence

René L. Schilling and Alexander Schnurr

The Symbol Associated with the Solution of a Stochastic Differential Equation

Henryk Zähle

Rates of almost sure convergence of plug-in estimates for distortion risk measures

Lorenz J. Schwachhöfer

Nonnegative curvature on disk bundles

Iuliu Pop and Ben Schweizer

Regularization schemes for degenerate Richards equations and outflow conditions

Guy Bouchitté and Ben Schweizer

Cloaking of small objects by anomalous localized resonance

Tom Krantz, Lorenz J. Schwachhöfer

Extrinsically Immersed Symplectic Symmetric Spaces

Alexander Kaplun

Continuous time Ehrenfest process in term structure modelling

Henryk Zähle

Ein aktuarielles Modell für die Portabilität der Alterungsrückstellungen in der PKV

Andreas Neuenkirch and Henryk Zähle

Asymptotic error distribution of the Euler method for SDEs with non-Lipschitz coefficients

Karl Friedrich Siburg, Pavel A. Stoimenov

Regression dependence

\section{Wilfried Hazod}

Continuous convolution hemigroups integrating a sub-multiplicative function

Sergio Conti and Ben Schweizer

On optimal metrics preventing mass transfer

Simon Castle, Norbert Peyerimhoff, Karl Friedrich Siburg

Billiards in ideal hyperbolic polygons

Ludwig Danzer

Quasiperiodic Tilings - Substitution Versus Inflation 
Direct simulation of the infinitesimal dynamics of semi-discrete approximations for convection-diffusion-reaction problems

2009-03 Franz Kalhoff and Victor Pambuccian

Existential definability of parallelism in terms of betweenness in Archimedean ordered affine geometry

2009-01 Henryk Zähle

Approximation of SDEs by population-size-dependent

Galton-Watson processes

2008-25 Winfried Hazod

Mehler semigroups, Ornstein-Uhlenbeck processes and background driving Lévy processes on locally compact groups and on hypergroups

2008-24 Karl Friedrich Siburg, Pavel A. Stoimenov

Symmetry of functions and exchangeability of random variables

2008-23 Ina Kirsten Voigt

Voronoi Cells of Discrete Point Sets

2008-22 Michael Lenzinger and Ben Schweizer

Effective reaction rates of a thin catalyst layer

2008-21 Michael Voit

Bessel convolutions on matrix cones: Algebraic properties and random walks

2008-20 Margit Rösler and Michael Voit

Limit theorems for radial random walks on $p \times q$-matrices as $p$ tends to infinity

2008-19 Michael Voit

Central Limit Theorems for Radial Random Walks on $p \times q$ Matrices for $p \rightarrow \infty$

2008-18 Michael Voit

Limit theorems for radial random walks on homogeneous spaces with growing dimensions

Sampling inspection by variables: nonparametric setting

2008-16 Guy Bouchitté and Ben Schweizer

Homogenization of Maxwell's equations with split rings

2008-15 Wilfried Hazod

Multiple selfdecomposable laws on vector spaces and on groups:

The existence of background driving processes 